\title{
Aeroelastic optimization of aircraft wings using a coupled three-dimensional panel- beam model
}

\section{Conlan-Smith, Cian; Andreasen, Casper Schousboe}

\author{
Published in: \\ AIAA Journal
}

Link to article, DOI:

10.2514/1.J059911

Publication date:

2021

Document Version

Peer reviewed version

Link back to DTU Orbit

Citation (APA):

Conlan-Smith, C., \& Andreasen, C. S. (2021). Aeroelastic optimization of aircraft wings using a coupled threedimensional panel-beam model. AIAA Journal, 59(4), 1374-1386. https://doi.org/10.2514/1.J059911

\section{General rights}

Copyright and moral rights for the publications made accessible in the public portal are retained by the authors and/or other copyright owners and it is a condition of accessing publications that users recognise and abide by the legal requirements associated with these rights.

- Users may download and print one copy of any publication from the public portal for the purpose of private study or research.

- You may not further distribute the material or use it for any profit-making activity or commercial gain

- You may freely distribute the URL identifying the publication in the public portal

If you believe that this document breaches copyright please contact us providing details, and we will remove access to the work immediately and investigate your claim. 


\title{
Aeroelastic Optimization of Aircraft Wings Using a Coupled Three-Dimensional Panel-Beam Model
}

\author{
Cian Conlan-Smith * and Casper Schousboe Andreasen ${ }^{\dagger}$ \\ Technical University of Denmark, DK-2800 Lyngby, Denmark.
}

\begin{abstract}
This work introduces an aeroelastic optimization framework with a coupled 3D panel method and Timoshenko beam finite element model. The method allows for optimization of both the exterior surface of the wing and interior structural properties. We investigate the effects of curved wall spars on the aeroelastic performance of converged designs, which have been shown to provide an improved performance due to the ability to create trade-offs between bending and torsional stiffness. Studies also highlight the importance of calculating aerodynamic loads in the deformed configuration and solving the coupled aeroelastic problem to convergence.
\end{abstract}

\section{Nomenclature}

$A=$ cross sectional area

A $=$ doublet aerodynamic influence coefficient

$a=$ flange thickness

B = source aerodynamic influence coefficient

$b \quad=$ wingspan

$C_{f}=\frac{f}{\frac{1}{2} \rho_{\infty} V_{\infty}^{2} c}$, normal load coefficient

$C_{m}=\frac{m}{\frac{1}{2} \rho_{\infty} V_{\infty}^{2} c^{2}}$, moment coefficient

$C_{P}=$ pressure coefficient

$c=$ chord length

$\bar{c}=\frac{\int_{0}^{\frac{b}{2}} c^{2} d y}{\int_{0}^{\frac{b}{2}} c d y}$, mean aerodynamic chord

$D=\operatorname{drag}$

d $=$ design variables

$E \quad=$ Young's modulus

$e \quad=$ spar center offset

$G \quad=$ shear modulus

*PhD student, Department of Mechanical Engineering, Section of Solid Mechanics, cicosm@ mek.dtu.dk. Member AIAA.

${ }^{\dagger}$ Associate Professor, Department of Mechanical Engineering, Section of Solid Mechanics. 


$$
\begin{aligned}
& h=\text { wingbox height } \\
& I_{x}=\text { second moment of area about local } x \text {-axis } \\
& I_{z} \quad=\text { second moment of area about local } z \text {-axis } \\
& \mathbf{K}=\text { stiffness matrix } \\
& K_{T}=\text { torsional stiffness } \\
& L \quad=\quad \text { lift } \\
& M_{p}=\text { pitching moment } \\
& m=\text { maximum relative camber } \\
& n=\text { normal vector } \\
& \mathbf{P}=\text { applied load vector } \\
& p=\text { relative position of maximum camber } \\
& S \quad=\quad \text { wing planform area } \\
& s \quad=\quad \text { spar thickness } \\
& \text { s }=\text { state variables } \\
& t=\text { maximum relative thickness } \\
& \mathbf{U}=\text { displacement vector } \\
& V \quad=\text { velocity } \\
& w \quad=\text { wingbox width } \\
& \alpha=\text { twist } \\
& \eta \quad=\frac{2 y}{b}, \text { normalized span location } \\
& \Lambda=\text { sweep angle } \\
& \mu \quad=\text { doublet strength } \\
& \Phi=\text { potential function } \\
& \psi \quad=\text { objective function } \\
& \rho \quad=\text { density } \\
& \sigma \quad=\quad \text { source strength } \\
& \sigma_{v}=\text { von Mises stress } \\
& \theta=\text { pitch angle } \\
& R=\frac{b^{2}}{S} \text {, aspect ratio }
\end{aligned}
$$

\section{Subscripts}

$a=$ associated with aerodynamic model

$b=$ associated with beam model 


\section{Introduction}

The application of multidisciplinary optimization (MDO) to the design of aircraft wings was first conducted in 1977 [1]. This study used gradient based optimization to demonstrate that such methods can be used to achieve designs with optimized trade-offs between weight and drag. Since then there has been a huge growth in the development of aeroelastic optimization methods and their application to a wide range of physics models with varying fidelity, from coupled lifting line-beam models [2] to large scale finite volume and finite element models [3 6]. Increasing fidelity of the models results in a large increase of computational expense which is of particular importance when applied to optimization problems as the physics model needs to be solved for each iteration of the optimization routine. To allow fast calculations of aerodynamic loads and structural deformations our focus is on low- to mid-range fidelity methods such as those based on potential flow theory.

Following their earlier work, Haftka et al [7, 8] later coupled a beam finite element model and vortex lattice method (VLM) with a profile drag correction obtained from experimental data. More recently, Jasa et al developed OpenAeroStruct, an open source aeroelastic framework that also uses a VLM-beam model based on Euler beams of tubular cross section [9]. The method was later adopted to model more practical wingbox configurations [10]. Dunning et al [11] applied a similar doublet lattice method with a 3D continuum mesh to the topology optimization of NASA's common research model (CRM) wing [12]. A higher fidelity option for the aerodynamics model is a 3D panel method, which has been used for MDO studies in the past. One such method was developed by Kennedy et al [13] which has been applied to a number of different studies with structural coupling to both shell [14, 15] and 3D continuum elements [16, 17]. Goetzendorf-Grabowski and Mieloszyk [18, 19] also implemented a coupled panel-shell framework for MDO.

The focus here is on MDO of aircraft wings, but it is important to note that the developed methods are also applicable to the design of other aeroelastic structures such as wind turbine blades. However, MDO studies of wind turbines in the literature commonly adopt models based on blade element momentum theory [20-23], usually coupled with beam models. Some exceptions include: Sessarego et al [24] who coupled a lifting line with a force-free wake and multi-body beam finite element method; and McWilliam et al [25, 26] who applied Lagrangian vortex dynamics to aero-structural MDO.

In structural optimization (SO), Aage et al [27] presented studies on topology optimization of the CRM wing under different loading conditions. The ultra-high resolution ( 1 billion finite elements) of this study allowed structural features to emerge that were unable to develop in previous lower resolutions studies due to a restricted design space. One such feature is curved wall spars which achieve an increased torsional stiffness at the cost of only slightly decreasing bending stiffness. 
The objective of the current work is to develop a coupled framework for solving aeroelastic optimization problems based on 3D panel methods and beam finite elements. These coupled models allow fast calculations of the physics of deformable wings with medium fidelity, and to our knowledge, have not been applied to MDO studies in the past. The coupled model expands upon a framework previously developed by the authors to conduct aerodynamic shape optimization of aircraft wings using 3D panel methods [28]. A newly derived generalized load-displacement transfer scheme is presented for coupled panel-beam methods. Design variables are used to control both the exterior shape of the wing and structural design of the interior. In this way, the optimizer has complete control over the external surface and internal domain of the wing, thus expanding the design space. A simple beam cross section parametrization is introduced in order to explore the effect of curved wall spars on the performance of optimized designs. Although the current parametrization is a simplification of the wing box structure we note that the method can be applied to a more advanced parametrization using cross sectional analysis tools such as VABS [29, 30] or BECAS [31].

The next section of this article introduces the reader to the panel-beam discretization and how the design is parametrized. Section III] outlines the physics modeling, which include: the 3D panel method; beam finite element model; the derivation of a generalized load-displacement transfer for coupled panel-beam models; and solution methods for the coupled system. The results section presents studies that explore the effect of introducing curved wall spars in the wingbox design with respect to SO for compliance minimization, SO and MDO for mass minimization with stress constraints, and MDO for drag minimization with stress constraints. Additionally, these studies will highlight the importance of calculating aerodynamic loads in the deformed configuration and the effect of sweep on optimized designs. Finally the findings are concluded in Section $\mathrm{V}$. Readers are referred to the appendices for a background discussion on the cross sectional stiffness of curved wall spars, details on stress calculations, and a derivation of sensitivity analysis.

\section{Discretization and parametrization}

\section{A. Discretization}

Fig. 1 shows the discretization of the problem using panels and beam finite elements. The surface of the wing is defined by a discrete set of airfoil sections which are assembled on a common axis corresponding to their quarter chord point. This quarter-chord axis is defined by the sweep angle $\Lambda$ from the $y-z$ plane, and lies in the $x-y$ plane (i.e. no dihedral) for cases where the wing geometry is optimized. Airfoil sections are defined parallel to the $x-z$ plane with a uniform spacing in the span-wise direction ( $y$-direction), refer to Fig. 11. The wing surface is discretized by quadrilateral panels which span the distance between airfoil sections. The root of the wing is at $y=0$ in the global coordinate system. Inside the wing, a single beam finite element also extends between any two neighboring airfoil sections. Beam nodes are located on the camber line of each airfoil section at the same fraction, $\kappa$, of the chord length, $c$ (refer to Fig. 1p). A beam element joins the nodes on consecutive airfoil sections and the orientation of the element is 
defined by a local coordinate system $\left(x_{b}, y_{b}, z_{b}\right)$. In the aerodynamic model symmetry is enforced by accounting for the influence from the mirror image of the wing about the $x-z$ plane, and the freestream flow is defined by the pitch angle, $\theta$, measured from the $z-y$ plane. For certain optimization problems a pitching moment constraint will be enforced about the center of gravity, $c g$. Typically the location of $c g$ is dependent on the entire aircraft and payload. In our examples $c g$ will be defined at the root airfoil midway downstream of the quarter chord axis, i.e. with the quarter chord axis starting at the origin and ending at the tip airfoil at a point $\left(x_{t}, y_{t}, 0\right), c g$ is defined to be at $\left(\frac{x_{t}}{2}, 0,0\right)$. This will correspond to modeling a flying wing configuration with an appropriately distributed payload.

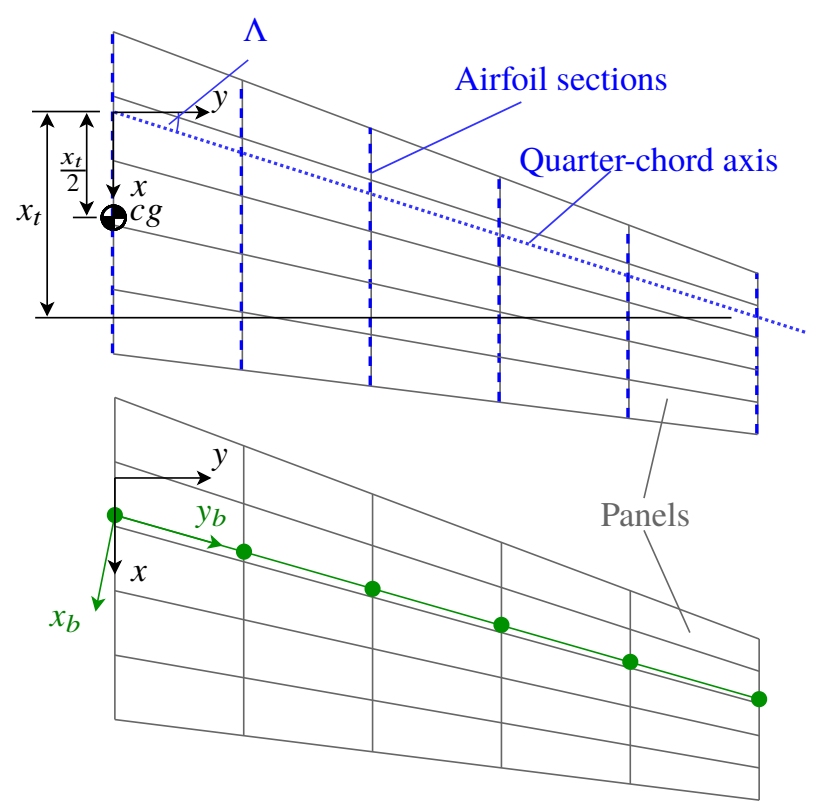

(a)

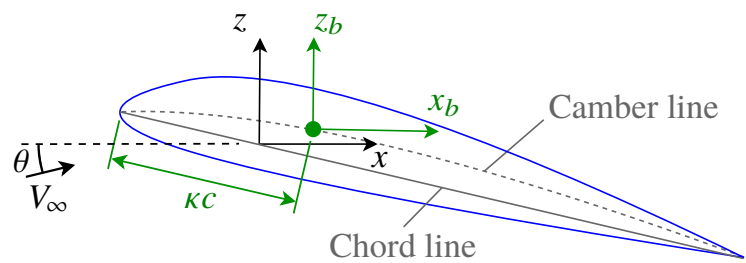

(b)

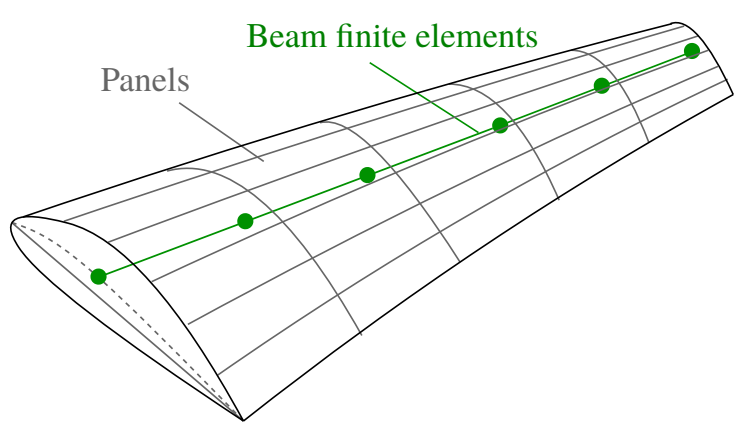

(c)

Fig. 1 Model discretization with airfoil parametrization shown in blue and beam finite elements shown in green: (a) planforms highlighting wing geometry and beam finite elements, (b) root airfoil section, and (c) 3D view.

\section{B. Airfoil parametrization}

For cases where the airfoil geometry is optimized we adopt the design variable description and airfoil parametrization introduced in [28]. Here, airfoils are defined based on a modified definition of the NACA 4 or NACA 5-digit airfoils. This parametrization uses up to five design variables per section to define an airfoil, namely, chord length $c$, maximum thickness $t$, maximum camber $m$, position of maximum camber $p$, and twist $\alpha$ (each labeled in Fig. 2). Airfoil design variables are filtered in the span-wise direction (as described in [28]) in order to avoid numerical artifacts.

\section{Beam parametrization}

In the structural model we assume that the load is carried only by the wing box which is defined by 5 design variables per section, as shown in Fig. 3 . The wing box of height, $h$, and width, $w$, comprises of forward and aft spars of equal 


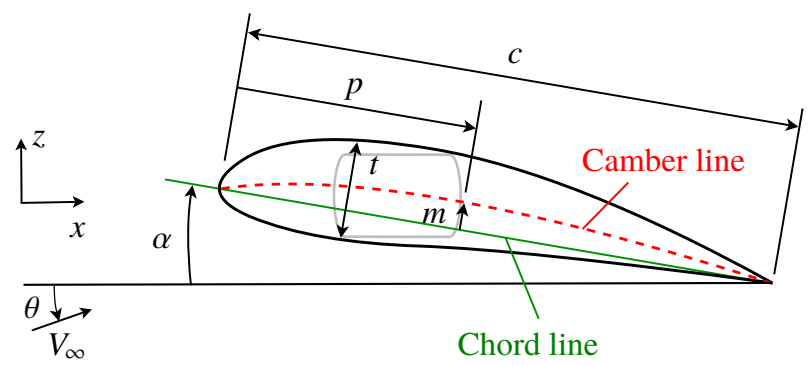

Fig. 2 Parametrization for airfoil sections based on NACA airfoil definitions, with maximum of 5 design variables: chord length $c$, maximum thickness $t$, maximum camber $m$, twist $\alpha$.

thickness, $s$, and upper and lower flanges of equal thickness, $a$. We include a fifth variable, $e$, which defines the center offset of the spar walls whose curved geometry is elliptic. The advantage of using ellipses (defined by major and minor axes) rather than circular arcs (defined by a radius and angle) is that the curves are less susceptible to roundoff errors when they approach a right-angled wingbox where the radius of a circular arc will grow to infinity and the angle tend to zero. The dimension, $w$, is between the centroids of the elliptic sections which means the internal area of the wingbox is kept roughly constant as the spar walls become increasingly more curved. This creates a fairer basis to compare straight and curved wall spars. The quantities $w, h$, and $e$ define the outer dimensions of the wingbox where thicknesses $a$ and $s$ are inset from the external wingbox geometry. Beam cross sections are parametrized in this manner at each airfoil section. The parametrization method is capable of tending from one extreme where spar walls are straight to the other extreme where the spar walls are semi-circular.

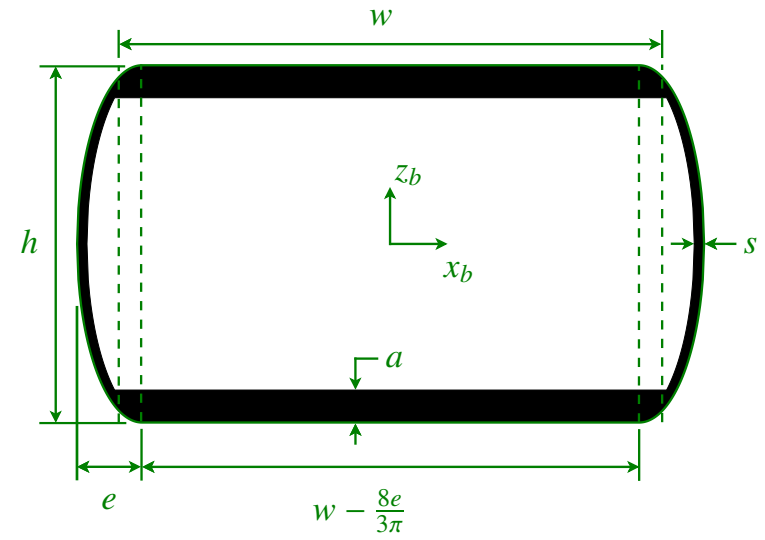

Fig. 3 Parametrization for a beam cross section based on 5 design variables: width $w$, height $h$, flange thickness $a$, spar thickness $s$, and spar center offset, $e$.

Upper bounds are placed on the design variables to ensure that the beam geometry is contained within the airfoil geometry. As the airfoil geometry is also defined by design variables these upper bounds are defined as fractions of the airfoil chord length and maximum thickness, e.g. $w=\bar{w} c$, where $\bar{w}$ is the design variable with bounds defined by 
$0.01 \leq \bar{w} \leq 0.4$. If the beam design variable is at the upper bound, the airfoil section geometry must be enlarged in order to increase the size of the beam. By defining the wingbox geometry in this manner the optimizer will know, for example, that $c$ must increase in order to increase $w$, as there will be a gradient with respect to $c$. By defining $w$ directly and setting the upper bound on $w$, no sensitivity information about the external geometry with respect to the internal geometry will be available to the optimizer.

The beam parameterization does not account for connections between the wingbox and the outer skin. At first this may seem somewhat unrealistic, however, the parameterization is robust enough to allow a large variance in stiffness properties whilst maintaining physical values. It should then be possible to reproduce the resulting stiffness properties using more conventional rib-spar structures.

\section{Physics modeling}

This section presents physics model of the coupled 3D panel and beam finite element methods. Two coupling methods are considered where aerodynamic loads can be calculated in either undeformed or deformed configurations. For the undeformed case the loads are calculated via the panel method and applied to the beam, where both models are solved only once. We will refer to this method as 1-way coupled. The second coupling method will be referred to as 2-way coupled, where displacements from the finite element model are used to perturb the panel geometry in the aerodynamic solver and the coupled problem is solved to convergence.

\section{A. Aerodynamic model}

The aerodynamic pressure loads are captured using constant source-doublet panels with a Prandtl-Glauert compressibility correction as introduced in [28]. We implement a Dirichlet boundary condition with a zero internal perturbation formulation defined by the governing equation

$$
\mathbf{R}_{a}(\boldsymbol{\mu}, \mathbf{U}, \mathbf{d})=\mathbf{A}(\mathbf{U}, \mathbf{d}) \boldsymbol{\mu}+\mathbf{B}(\mathbf{U}, \mathbf{d}) \boldsymbol{\sigma}(\mathbf{U}, \mathbf{d})=\mathbf{0}
$$

where $\mathbf{A}$ and $\mathbf{B}$ are the aerodynamic influence coefficients which depend on the design variables, $\mathbf{d}$, and (for 2-way coupling) the solution to the finite element problem, $\mathbf{U}$. Equation (1) is used to solve for the doublet strengths, $\boldsymbol{\mu}$, with the source strengths are defined as

$$
\sigma_{i}=\boldsymbol{V}_{\infty} \cdot \boldsymbol{n}_{i}
$$

where $\boldsymbol{V}_{\infty}$ is the freestream velocity and $\boldsymbol{n}_{i}$ is the outward pointing normal of panel $i$. Once the doublet strengths are known the local velocity across a panel $i$, can be calculated as the gradient of the potential function, $\Phi$, which simplifies to

$$
\boldsymbol{V}_{i}=\boldsymbol{\nabla} \Phi_{i}=\left(\frac{d \mu}{d \bar{x}}, \frac{d \mu}{d \bar{y}}, 0\right)_{i}^{T}+\left(\boldsymbol{V}_{\infty} \cdot \overline{\boldsymbol{x}}, \boldsymbol{V}_{\infty} \cdot \overline{\boldsymbol{y}}, 0\right)_{i}^{T}
$$


where $\bar{x}$ and $\bar{y}$ are local panel coordinates whose axes are define by unit vectors $\overline{\boldsymbol{x}}$ and $\overline{\boldsymbol{y}}$ in the global reference frame. Local velocity is then used to calculate the pressure coefficient via the Bernoulli equation

$$
C_{P, i}=1-\frac{\left\|V_{i}\right\|^{2}}{\left\|V_{\infty}\right\|^{2}}
$$

The total induced drag and lift are calculated using a far-field approximation via a Trefftz-plane integration as it has been found to be more numerically stable for optimization problems compared with $C_{P}$-integration [28]. Trefftz-plane integration is calculated as [32]

$$
\begin{gathered}
D=-\frac{1}{2} \rho_{\infty} \sum_{i=1}^{N_{w}} \mu_{i} l_{i} \boldsymbol{v}_{i} \cdot \boldsymbol{n}_{i} \\
L=\rho_{\infty} V_{\infty} \sum_{i=1}^{N_{w}} \mu_{i} \cos \left(\phi_{i}\right) l_{i}
\end{gathered}
$$

where $N_{w}$ is the number of wake panels, $l_{i}$ and $\phi_{i}$ are the length and orientation of wake panel $i$ in the Trefftz-plane. Because panel methods assume inviscid and incompressible flow, the induced drag is the only type of drag captured by this model. It is possible to couple these methods to viscous solvers [33] but this will require prior knowledge of the boundary layer shape which is difficult to predict when airfoils are free to change during the optimization procedure. As a result, only the induced drag is considered in this work. A fixed wake model is used whose shape is achieved by projecting the trailing edge downstream in the free-stream direction.

\section{B. Structural model}

The internal structure of the wing is modeled using one-dimensional cubic beam finite elements. Element degrees of freedom (DOF) and local loads are shown in Fig. 4

Aerodynamic loads calculated in the panel method are transferred to beam nodes in form of a load vector, $\mathbf{P}$, which consists of local forces, $\boldsymbol{F}$, and moments, $\boldsymbol{M}$. These loads are dependent on both the design variables, $\mathbf{d}$, and the solution to the panel method, $\boldsymbol{\mu}$. Only aerodynamic loads are applied, where self-weight is not considered. We note that by neglecting self-weight the loading will be slightly higher as the wing's weight would impose a force counteracting the lifting loads, thus potentially reducing the stresses. The global vector of nodal displacements and rotations, $\mathbf{U}$, is determined via the governing equation for the beam,

$$
\mathbf{R}_{b}(\boldsymbol{\mu}, \mathbf{U}, \mathbf{d})=\mathbf{K}(\mathbf{d}) \mathbf{U}-\mathbf{P}(\boldsymbol{\mu}, \mathbf{U}, \mathbf{d})=\mathbf{0}
$$

It is noted that for 2-way coupling, $\mathbf{P}$ is also dependent on $\mathbf{U}$ as the aerodynamic loads are calculated in the deformed configuration. The global stiffness matrix, $\mathbf{K}$, is a function of $\mathbf{d}$, and formed from assembling element stiffness matrices, 


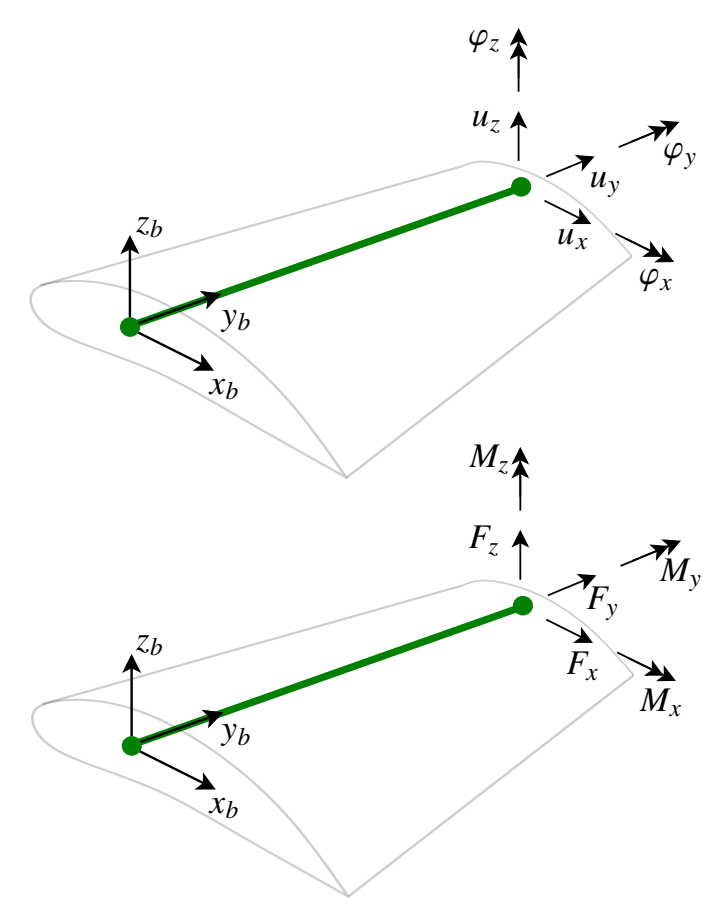

Fig. 4 A beam finite element with local coordinate system, degrees of freedom and applied loads: displacements, $\left\{u_{x}, u_{y}, u_{z}\right\}$; rotations, $\left\{\phi_{x}, \phi_{y}, \phi_{z}\right\}$; conjugate forces, $\left\{F_{x}, F_{y}, F_{z}\right\}$; and applied moments, $\left\{M_{x}, M_{y}, M_{z}\right\}$.

$\mathbf{K}_{e}$ via

$$
\mathbf{K}_{e}(\mathbf{d})=\int_{0}^{l_{e}} \mathbf{T}(\mathbf{d})^{\mathrm{T}} \mathbf{B}^{\mathrm{T}} \mathbf{K}_{c s}(\mathbf{d}) \mathbf{B} \mathbf{T}(\mathbf{d}) d l
$$

where $\mathbf{T}$ is a rotation matrix accounting for the orientation of the beam in the global coordinate system (refer to Fig. 1). By evaluating the integral in (8) using gauss quadrature, one can account for a varying cross section along the element. The constitutive model for a cross section of the beam is based on an axial model, thin-walled torsional model, and Timoshenko model in bending. Only doubly symmetric cross sections and isotropic materials are considered in this work and as such the constitutive stiffness matrix, $\mathbf{K}_{c s}$, is a diagonal matrix with the following non-zero entries

$$
K_{c s, 11}=G A, K_{c s, 22}=E A, K_{c s, 33}=G A, K_{c s, 44}=E I_{x}, K_{c s, 55}=G K_{T}, K_{c s, 66}=E I_{z}
$$

From the dimensions shown in Fig. 3 analytical expressions for area and second moments of area can be derived using the principles of superposition and the parallel axis theorem. These are standard methods which are well documented [34] and as final expressions are long they are not presented here. Note that the shear correction factors in (9) have been neglected as they are not readily available for all curved wall spar geometries. However, as these values are typically higher for curved cross sections, we suspect that including shear factors would increase the incentive for the optimizer to create curved wall spars. If the reader is interested in achieving shear correction factors for a given wingbox with curved wall spars we recommend using VABS [29, 30] or BECAS [31] cross-sectional analysis tools. 
The matrix $\mathbf{B}$ in (8) is the strain displacement matrix defined such that the strains and curvatures can be achieved by

$$
\left\{\begin{array}{l}
\boldsymbol{\varepsilon} \\
\boldsymbol{\kappa}
\end{array}\right\}=\mathbf{B U}_{e}=\left[\mathbf{N}^{\prime}+S \mathbf{N}\right] \mathbf{U}_{e}
$$

where $\mathbf{N}$ are the shape functions, whose derivatives are denoted by the prime, and $S$ is used to account for rotations of

the cross section in calculating the strains due to bending [35], i.e. $\varepsilon_{x}=\frac{d u_{x}}{d l}+\varphi_{z}$ and $\varepsilon_{z}=\frac{d u_{z}}{d l}-\varphi_{x}$ (refer to DOF in Fig. 4).

\section{Load-displacement transfer}

The beam finite element model assumes sections remain planar and undergo rigid body displacements and rotations. Since nodes of an airfoil section on the panel mesh lie in-plane with a single node on the beam mesh, the perturbations of a single aerodynamic node, $\delta \boldsymbol{X}_{a}$, is described using displacements and rotations of the beam node at the same span-wise location. Assuming small rotations the displacement transfer can be defined as

$$
\delta \boldsymbol{X}_{a}=\delta \boldsymbol{u}_{b}-\boldsymbol{r} \times \delta \boldsymbol{\varphi}_{b}=\delta \boldsymbol{u}_{b}+\delta \boldsymbol{\varphi}_{b} \times \boldsymbol{r}
$$

where $\delta \boldsymbol{u}_{b}$ and $\delta \varphi_{b}$ are displacements and rotations at the beam node, and $\boldsymbol{r}$ is a spatial vector from the beam node to the aerodynamic node.

Aerodynamic forces are transferred to the beam in form of distributed loads $\mathbf{f}$ and $\mathbf{m}$. The total load acting on an element is achieved integrating distributed loads from along the element and summing the contribution from each of the $N$ panels at the same span-wise section.

$$
\boldsymbol{P}_{e}=\left\{\begin{array}{c}
\boldsymbol{F} \\
\boldsymbol{M}
\end{array}\right\}=\sum_{i=1}^{N} \int_{0}^{l_{e}}\left\{\begin{array}{c}
\mathbf{f} \\
\mathbf{m}
\end{array}\right\} d l
$$

To ensure consistency it is required that the total force calculated in the aerodynamic model matches the applied load in the beam model. To ensure conservativeness the virtual work performed by the structural load undergoing virtual displacements and rotations must be equal to the virtual work of the panel method [36]. Using this requirement we can derive a consistent and conservative load-displacement transfer method. The virtual work of a beam element loaded by a single panel (as shown in Fig. 5) is defined as

$$
\delta W_{b}=\int_{0}^{l_{e}}\left\{\begin{array}{l}
\mathbf{f} \\
\mathbf{m}
\end{array}\right\}^{\mathrm{T}}\left\{\begin{array}{l}
\delta \boldsymbol{u} \\
\delta \boldsymbol{\varphi}
\end{array}\right\} d l=\int_{0}^{l_{e}}\left(\mathbf{f}^{\mathrm{T}} \delta \boldsymbol{u}+(\boldsymbol{r} \times \mathbf{f})^{\mathrm{T}} \delta \boldsymbol{\varphi}\right) d l
$$

where the applied moments arise from translating the force from the panel to the beam element meaning that $\mathbf{m}=\boldsymbol{r} \times \mathbf{f}$, with $\boldsymbol{r}$ being a spatial vector from the beam element to the panel. Manipulating the second term in the integrand by 
$(\boldsymbol{r} \times \mathbf{f})^{\mathrm{T}} \delta \boldsymbol{\varphi}=\delta \boldsymbol{\varphi}^{\mathrm{T}}(\boldsymbol{r} \times \mathbf{f})=(\delta \boldsymbol{\varphi} \times \boldsymbol{r})^{\mathrm{T}} \mathbf{f}=\mathbf{f}^{\mathrm{T}}(\delta \boldsymbol{\varphi} \times \boldsymbol{r})$, yields

$$
\delta W_{b}=\int_{0}^{l_{e}} \mathbf{f}^{\mathrm{T}}(\delta \boldsymbol{u}+\delta \boldsymbol{\varphi} \times \boldsymbol{r}) d l
$$

The virtual work of the aerodynamic model by a single panel is

$$
\delta W_{a}=\int_{S} q_{\infty} C_{P} \boldsymbol{n}^{\mathrm{T}} \delta \boldsymbol{X}_{a} d S
$$

The panel method assumes that $C_{P}$ is constant over the panel and each panel is flat meaning that $\boldsymbol{n}$ is also constant over the panel. Additionally, we assume perturbations in the chord-wise direction of the panel are constant. Using equation (11) and representing $d S$ as $w d l$ we find that

$$
\delta W_{a}=q_{\infty} C_{P} J_{l} \boldsymbol{n}^{\mathrm{T}} \int_{0}^{l_{e}} w(\delta \boldsymbol{u}+\delta \boldsymbol{\varphi} \times \boldsymbol{r}) d l
$$

where $J_{l}$ is a linear mapping introduced to account for cases where the length of the panel is not equal to the element length, $l_{e}$ (refer to Fig. 5). By equating equations (14) and (16), the distributed load, $\mathbf{f}$ from a single panel can be defined as

$$
\mathbf{f}=q_{\infty} C_{P} J_{l} w \boldsymbol{n}
$$

The applied load can now be calculated by inserting (17) and $\mathbf{m}=\boldsymbol{r} \times \mathbf{f}$ into (12).

\section{2-way coupled solution method}

The coupled problem can be solved using fixed point iterations in which the panel method is solved in the undeformed configuration and calculated loads are used to solve the beam problem. The displacements from the beam problem are then passed back to the panel method and loads are calculated in the deformed configuration. This process continues until the change in state variables, $\mathbf{s}=\{\boldsymbol{\mu} \mathbf{U}\}^{\mathrm{T}}$, between iterations is less than the tolerance $\epsilon$.

Alternatively a Newton method is used where the residual $\mathbf{R}=\left\{\mathbf{R}_{a} \mathbf{R}_{b}\right\}^{\mathrm{T}}$ is calculated on each iteration and a solution step, $\delta \mathbf{s}$, is calculated using the Jacobian $\frac{\partial \mathbf{R}}{\partial \mathbf{s}}$, as

$$
\delta \mathbf{s}={\frac{\partial \mathbf{R}^{-1}}{\partial \mathbf{s}}}^{\mathbf{R}}
$$

where

$$
\frac{\partial \mathbf{R}}{\partial \mathbf{S}}=\left[\begin{array}{ll}
\frac{\partial \mathbf{R}_{a}}{\partial \boldsymbol{\mu}} & \frac{\partial \mathbf{R}_{a}}{\partial \mathbf{U}} \\
\frac{\partial \mathbf{R}_{b}}{\partial \boldsymbol{\mu}} & \frac{\partial \mathbf{R}_{b}}{\partial \mathbf{U}}
\end{array}\right]=\left[\begin{array}{cc}
\mathbf{A} & {\left[\frac{\partial \mathbf{A}}{\partial \mathbf{U}} \boldsymbol{\mu}+\frac{\partial \mathbf{B}}{\partial \mathbf{U}} \boldsymbol{\sigma}+\mathbf{B} \frac{\partial \sigma}{\partial \mathbf{U}}\right]} \\
-\frac{\partial \mathbf{P}}{\partial \boldsymbol{\mu}} & {\left[\mathbf{K}-\frac{\partial \mathbf{P}}{\partial \mathbf{U}}\right]}
\end{array}\right]
$$




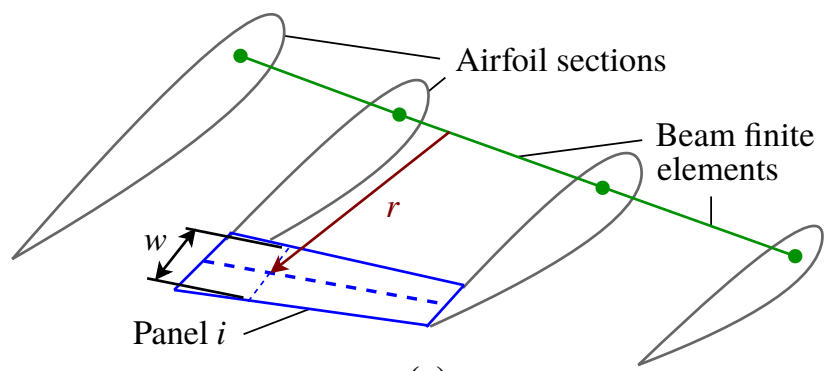

(a)

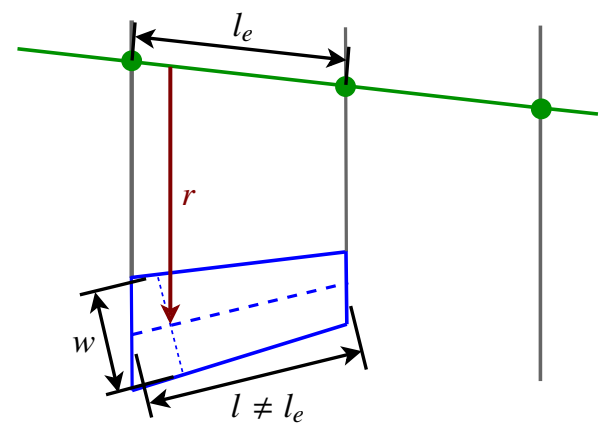

(b)

Fig. 5 Schematic of load displacement transfer scheme from a single panel in (a) 3D and (b) planform views.

The solution is terminated once the residual norm is less than the tolerance $\epsilon$.

Fixed point iterations generally take more iterations to converge as it is a first order convergence scheme and the Newton method is second order. However, a fixed point iteration method does not require the user to calculate the Jacobian matrix, which for the panel method can take up to 8 times the computational cost of the forward problem [28]. This means fixed point iterations generally converge to a solution faster than the Newton method with respect to wall-clock time. However, the Newton method is more robust for compliant wings where deformations are large [9]. Generally, we use fixed point iterations due to the reduced computational cost, and a value of $\epsilon=10^{-3}$ was used for optimization problems as it was found to be a good trade-off between accuracy and computational time.

\section{Results}

The results section is divided into two subsections. The first subsection, (A), contains studies where the internal structure of the CRM wing is optimized for compliance minimization and stress-constrained mass minimization. The goal of these studies is to provide the reader with insight into the beam parametrization, coupling procedure, and problem formulation. Subsection (B) details multidisciplinary shape optimization studies where both the external wing geometry and the internal beam structure is parameterized by design variables. Each problem is solved for a freestream velocity of Mach 0.4 using 4000 panels and 40 beam finite elements. All optimization problems are solved using the method of moving asymptotes [37] where gradients are calculated using adjoint sensitivity analyses. Readers are referred to appendix C for derivations of gradients. 


\section{A. Structural optimization of the CRM wing}

As a first study we investigate a fixed skin CRM wing optimization with design variables, $\mathbf{d}=\{\boldsymbol{w}, \boldsymbol{h}, \boldsymbol{a}, \boldsymbol{s}, \boldsymbol{e}\}$. The objective is to minimize compliance subject to a volume constraint, as defined in [20], where compliance is summed for multi-load cases with each case weighted equally, and the volume fraction is defined as the volume of the beam divided by the total volume of the wing. This is a similar formulation to the topology optimization study presented in [27] where curved wall spars appeared. Here, the problem is solved using only 1-way coupling (i.e. in the undeformed configuration). Studies are presented with pitch angles, $\theta=\left\{0^{\circ}, 5^{\circ}, 10^{\circ}\right\}$. The beam nodes are positioned at the $40 \%$ of the chord length in each section, as shown in Fig. 6a The primary loading of the beam in each case is given in Figs. $6 \mathrm{~b}$ and $6 \mathrm{c}$, where coefficients are calculated using the mean aerodynamic chord.

$$
\begin{array}{ll}
\min _{\mathbf{d} \in \mathbb{R}^{N_{\mathrm{d}}}} & : \psi=\sum_{\theta} C_{\theta}(\mathbf{U}, \mathbf{d}) \\
\text { subject to } & : V(\mathbf{d})-\bar{V} \leq 0 \\
& \underline{d} \leq d_{i} \leq \bar{d} \quad \text { for } i=1, \ldots, N_{\mathrm{d}}
\end{array}
$$

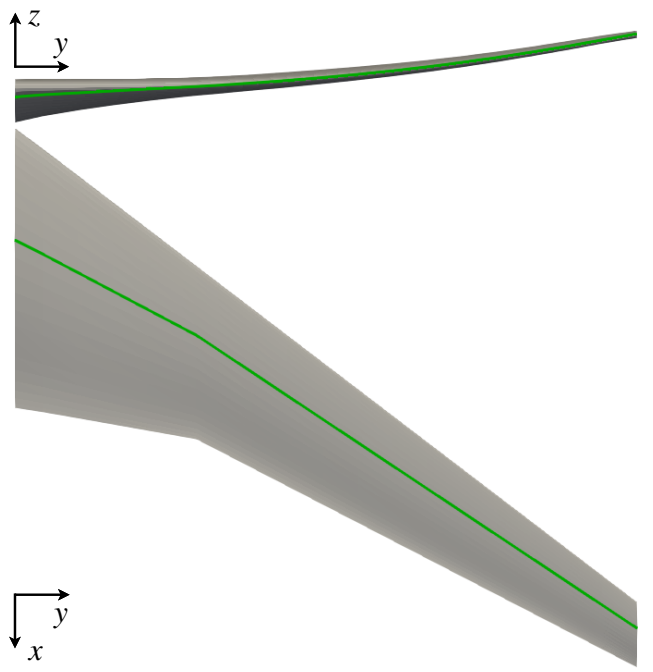

(a)

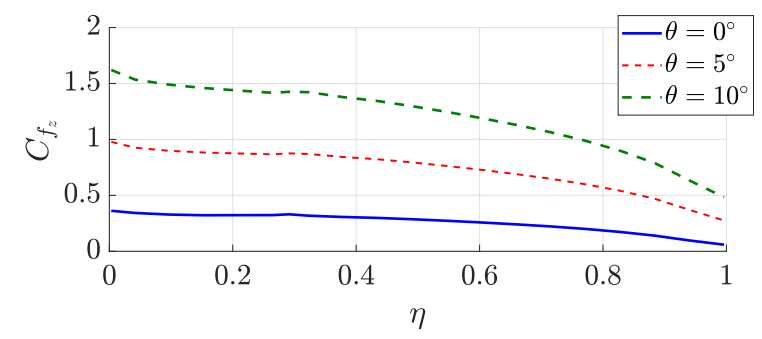

(b)

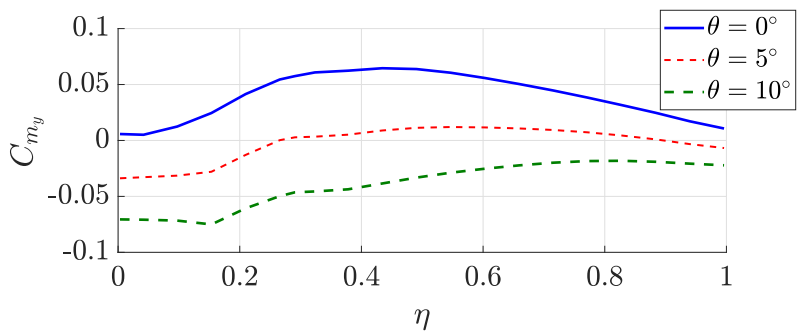

(c)

Fig. 6 Undeformed CRM wing geometry and primary beam loading at different pitch angles: (a) plan and elevation of the CRM wing with beam axis shown in green, (b) normal load coefficient, (c) torsional load coefficient.

The optimization problem is solved where the spar walls are straight, i.e. $\boldsymbol{e}=\mathbf{0}$ in Fig. 3 , and where curved wall spars are allowed to form, i.e. $\boldsymbol{e}$ is a design variable, in order to investigate possible gains. The relative difference in the objective function between optimized designs with fixed straight wall spars and when curved wall spars are allowed to form is shown in Fig. 7 , where $\Delta_{C}=\frac{\psi_{e=0}-\psi_{e}}{\psi_{e=0}}$. The results show a reduction in compliance with the curved wall 


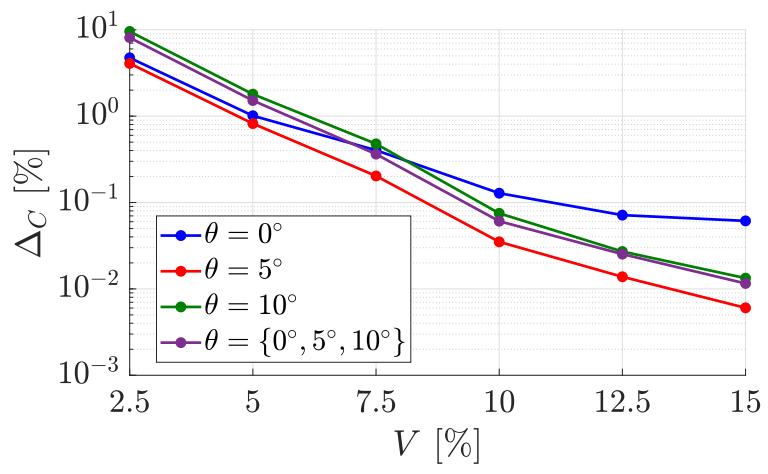

Fig. 7 Reduction in compliance when curved wall spars are allowed for different volume fractions.

spars for a range of volume fractions, $\bar{V}$. The reduction in compliance is due to an increased torsional stiffness of the curved wall spars. However, this increase in torsional stiffness corresponds to a reduction in bending stiffness (refer to appendix A for an example study). As the volume fraction of the structure increases the optimizer is less likely to use curved wall spars and the performance of the two designs are similar. This is because the thicker-walled box sections already provide adequate torsional stiffness, as can be seen in the definition of $K_{T}$ (appendix A, equation 26). On the other hand when tight restrictions are placed on the available material the use of curved wall spars become more advantageous, with reductions in compliance of up to $10 \%$.

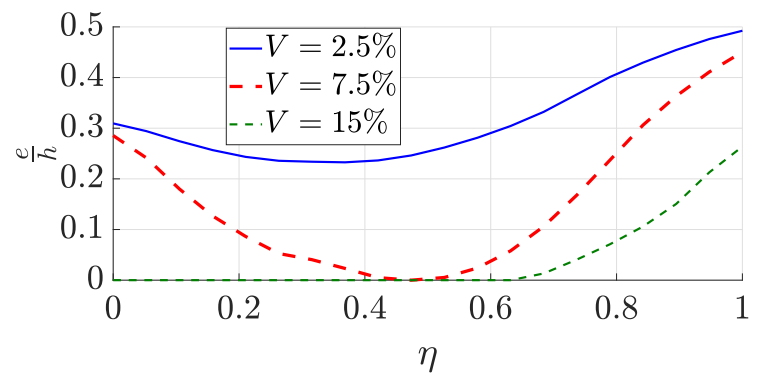

Fig. 8 Span-wise variation in curvature of the spar walls for the $\theta=\left\{0^{\circ}, 5^{\circ}, 10^{\circ}\right\}$ case with different volume fractions. Spar walls are straight for $\frac{e}{h}=0$ and semi-circular for $\frac{e}{h}=0.5$.

Fig. 8 plots the ratio of $e$ to $h$ along the span of the wing for different volume fractions in the multi-load case. The ratio of $e$ to $h$ describes how curved the spar walls are, where $\frac{e}{h}=0$ corresponds to straight wall spars and $\frac{e}{h}=0.5$ to semi-circular spar walls. Curved wall spars form mainly around the tip and root of the wing, and are less likely to appear for higher volume fractions as previously discussed. Cross sections of wing and beam at the root, mid-section, and tip for a selection of volume fractions are shown in Fig. 9. From these sections it is clear to see the straight and curved wall spar designs tend to converge for higher volume fractions. For low volume fractions the beam is wider in the mid-sections of the wing than the root or tip. This increases the torsional stiffness away from the root where is it not possible to increase the height due to the restrictions of the airfoil section. In this case the torsional stiffness peaks 
around the Yehudi break, whereas for larger volume fractions the largest torsional stiffness is at the root, corresponding to the largest airfoil section. Differences in the performance of these structures may not be intuitively obvious from the cross sections alone. A more informative way to compare the differences in design is to look at the distribution along the wing of the sectional stiffness properties as shown in Fig. 10 Here the sectional stiffness is normalized with wing's planform area $S$, span $b$, and mean aerodynamic chord $\bar{c}$. These distributions show that by introducing curved wall spars the optimizer can sacrifice bending stiffness for an increase in torsional stiffness. This is most evident for low volume fractions as designs converge for when the volume fraction increases.

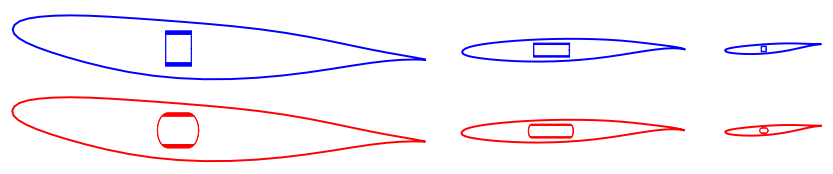

(a) $V=2.5 \%$

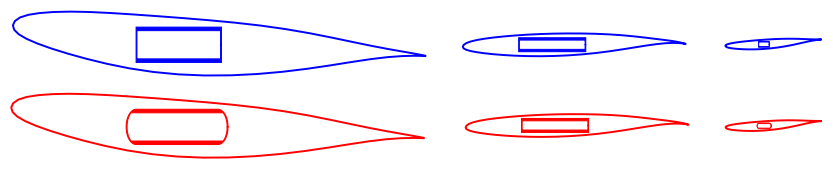

(b) $V=7.5 \%$

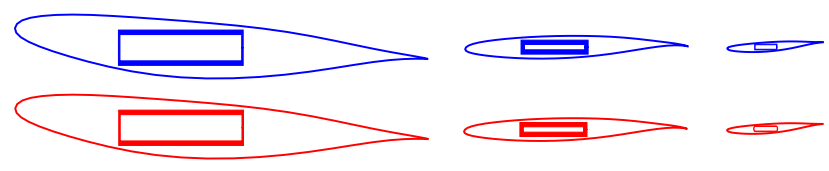

(c) $V=15 \%$

Fig. 9 Airfoil and beam sections for $\theta=\left\{0^{\circ}, 5^{\circ}, 10^{\circ}\right\}$ case with different volume fractions. Root, mid, and tip sections from left to right with straight wall spar designs are shown on top, curved wall spars designs are shown below.

Comparing to the topology optimized designs presented in [27], the spars have a higher curvature in our case. However, it is important to note that the airfoil skin thickness is included in topology optimized designs which will increase the torsional stiffness. As discussed earlier, one may argue the current beam parametrization is non-physical as the connection between the wingbox and airfoil skin is not considered. However, the simplified model is adequate for demonstrating the differences in performance of curved wall spar structures. Additionally, the parametrization allows stiffness distributions to be optimized in a manner that is robust and produces physical values. It can then be possible to realize these stiffness distributions using more traditional rib-spar structures post-optimization.

When dimensions of the wingbox are free to change, as in the previous example, it can be difficult to demonstrate the trade-offs between straight and curved wall spars as the wingbox dimensions have a large impact on the stiffness properties of the beam (refer to appendix A). It is more clearly demonstrated by fixing the wingbox dimensions and solving 20] again with only one design variable per section to control the shape of the spars, i.e. $\mathbf{d}=\boldsymbol{e}$. All other wingbox dimensions are defined by the optimized design with straight wall spars in the previous problem. Fig. 11 shows the difference in sectional bending and torsional stiffness along the wing for the multi-load case, $\theta=\left\{0^{\circ}, 5^{\circ}, 10^{\circ}\right\}$. 


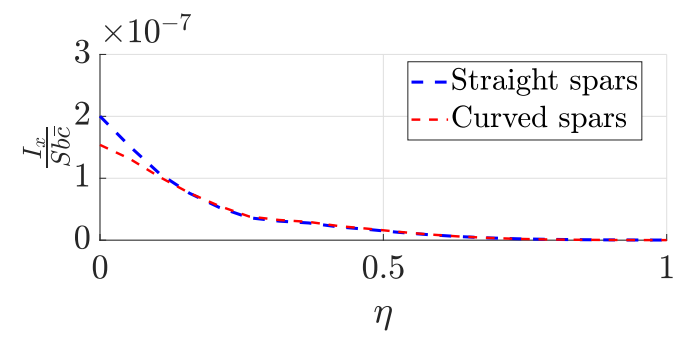

(a) $\bar{V}=2.5 \%$, bending stiffness

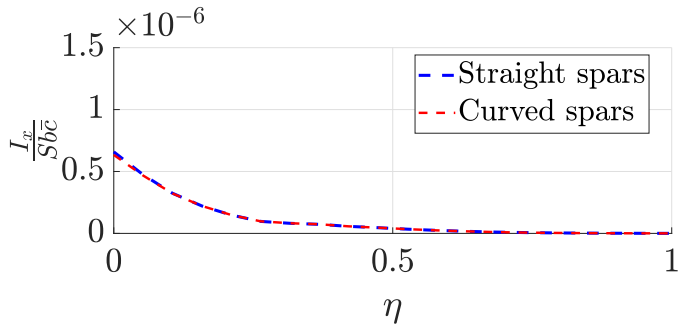

(c) $\bar{V}=7.5 \%$, bending stiffness

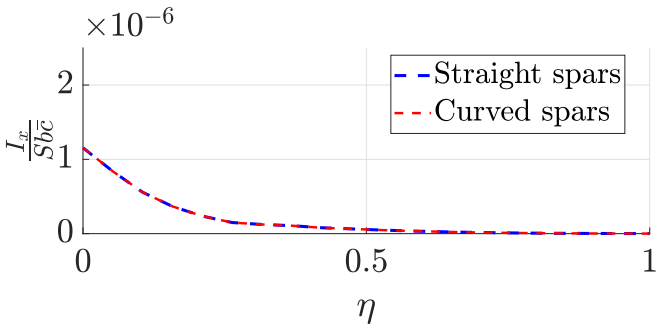

(e) $\bar{V}=15 \%$, bending stiffness

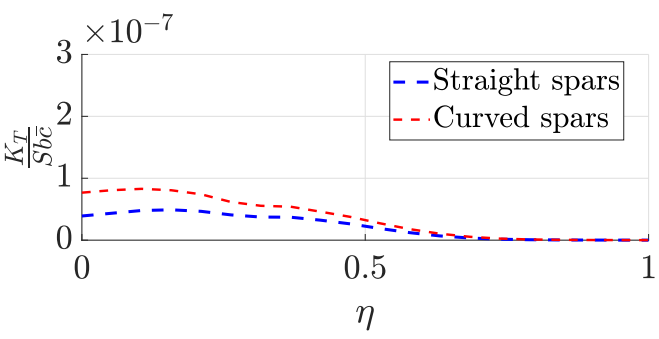

(b) $\bar{V}=2.5 \%$, torsional stiffness

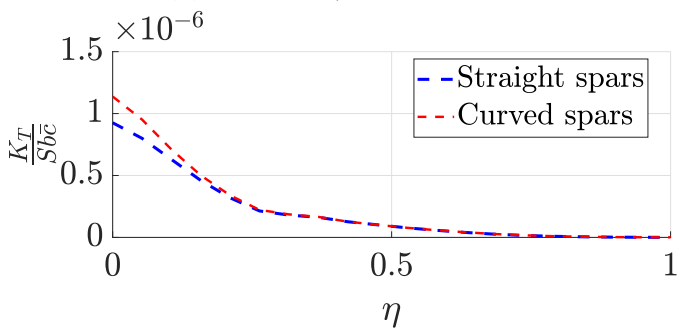

(d) $\bar{V}=7.5 \%$, torsional stiffness

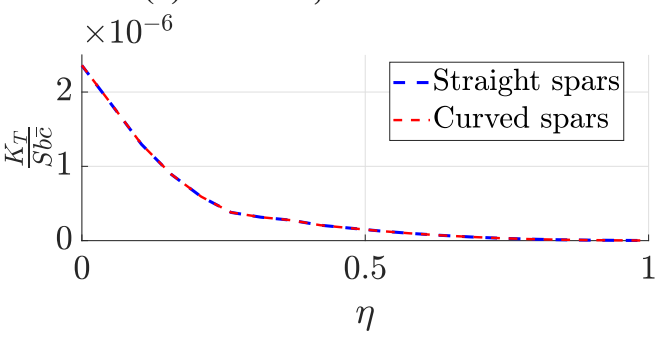

(f) $\bar{V}=15 \%$, torsional stiffness

Fig. 10 Stiffness properties of converged designs for primary loading in multi-load case, $\theta=\left\{0^{\circ}, 5^{\circ}, 10^{\circ}\right\}$. Solved for different volume fractions, $\bar{V}$, with both straight and curved wall spars.

For these designs the optimizer can curve the spar walls leading to increases in torsional stiffness at the cost of reducing bending stiffness. This clearly shows the freedom of the parametrization to create optimized trade-offs between torsional and bending stiffness. As the spars become thicker, creating curved wall spars becomes less advantageous, and for a volume fraction, $V \geq 15 \%$, the spar walls remain straight. Curved wall spars mainly form towards the tip of the wing and in some cases at the root. This makes sense as at the tip of the wing the bending strain tends to zero but the torsional strain does not. For low volume fractions allowing curved wall spars can lead to an increase in torsional stiffness of over $10 \%$.

Compliance minimization is the standard problem formulation for structural optimization problems. However, it is not uncommon that aircraft wings are designed to experience large deformations, and by minimizing compliance the deflections are minimized. A more natural formulation for structural optimization of an aircraft wing would be to 


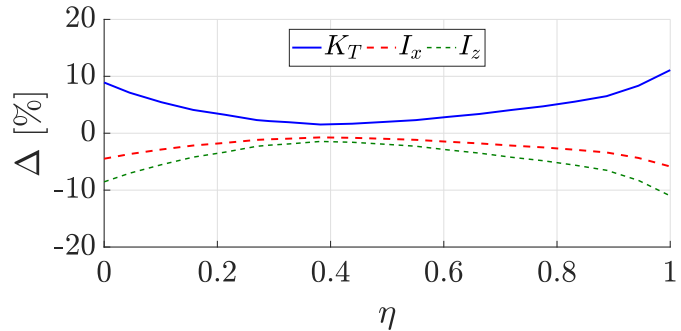

(a) $\bar{V}=2.5 \%$

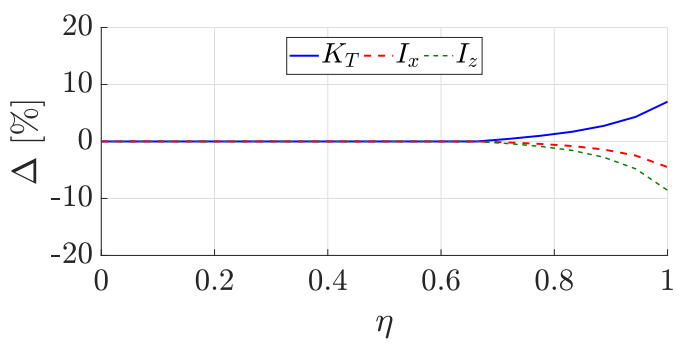

(c) $\bar{V}=7.5 \%$

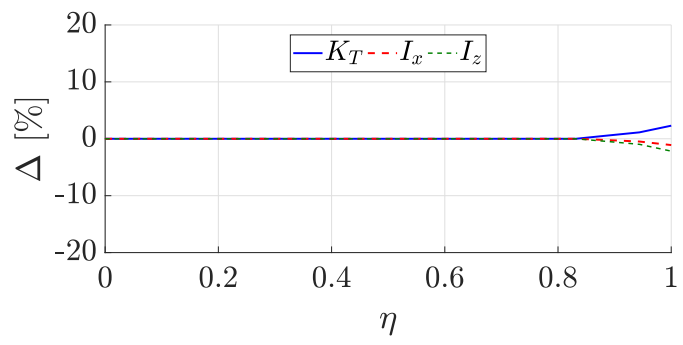

(e) $\bar{V}=12.5 \%$

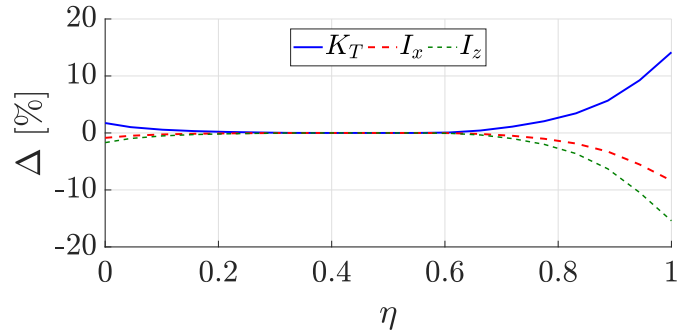

(b) $\bar{V}=5 \%$

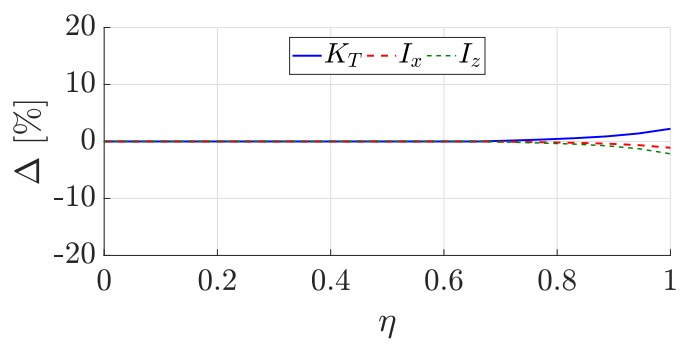

(d) $\bar{V}=10 \%$

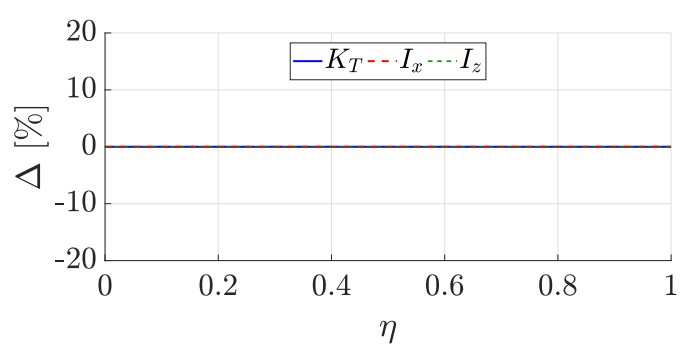

(f) $\bar{V}=15 \%$

Fig. 11 Difference in sectional stiffness between curved and straight wall spars for different bounds on volume fraction, $\bar{V}$, and only one design variable, $e$.

minimize mass subject to stress constraints, defined as

$$
\begin{aligned}
& \min _{\mathbf{d} \in \mathbb{R}^{N_{\mathrm{d}}}} \quad: m(\mathbf{d}) \\
& \text { subject to }: \frac{\hat{\sigma}_{i}(\mathbf{U}, \mathbf{d})}{\bar{\sigma}}-\beta \leq 0 \text { for } i=1, \ldots, N_{r} \\
& \underline{d} \leq d_{i} \leq \bar{d} \quad \text { for } i=1, \ldots, N_{\mathrm{d}}
\end{aligned}
$$

where $\hat{\sigma}_{i}$ is the maximum stress in region $i, \bar{\sigma}$ is the yield/ultimate stress, and $\beta$ is a fixed scalar which introduces a safety factor. A p-norm function is used to approximate the maximum stress and aggregate the stresses into $N_{r}$ regional constraints by

$$
\max \left(\sigma_{v}\right) \simeq \hat{\sigma}=c_{I} \sigma_{p n}=c_{I}\left(\sum_{i} \sigma_{v, i}^{p}\right)^{\frac{1}{p}}
$$

where $c_{I}$ is a scaling parameter used to ensure the p-norm correctly approximates the maximum von Mises stress and is 
defined as [38]

$$
c_{I}=\zeta \frac{\max \left(\sigma_{v}\right)_{I-1}}{\sigma_{p n, I-1}}+(1-\zeta) c_{I-1}
$$

where $I$ is the design iteration number and $\zeta$ is taken to be 0.5 throughout. The von Mises stress, $\sigma_{v}$ is calculated at the gauss points, at 16 locations on the cross section as described in appendix B Stress constraints are enforced on $N_{r}$ regions, to improve the performance of the p-norm approximation. These regions are defined based on span-wise location where the beam is divided into $N_{r}$ equal segments. Typically, $N_{r}=20$ was used with 40 finite elements and 4 gauss points along the element, leading to a total of 128 stress calculations per region.

Here, the problem is solved using both 1- and 2-way coupling methods. The CRM wing is again taken as the fixed outer geometry. For $\beta=1$, curved wall spars achieve a reduction in mass of 13-14\% for both 1- and 2-way coupled problems. The reasoning for this is the same as previously discussed for the compliance minimization problem: the curved wall spar parametrization is better able to create optimized trade-offs between torsional stiffness and bending stiffness. The maximum stress ratio in each section along the wing is shown in Fig. 12 for different values of $\beta$. The changes in mass when going from 1-way coupling to 2-way coupling ( $\left.\Delta_{m}=\frac{m_{2}-m_{1}}{m_{1}}\right)$ is shown in Table 1 and the stiffness distributions for straight wall spar designs are shown in Fig. 13 .

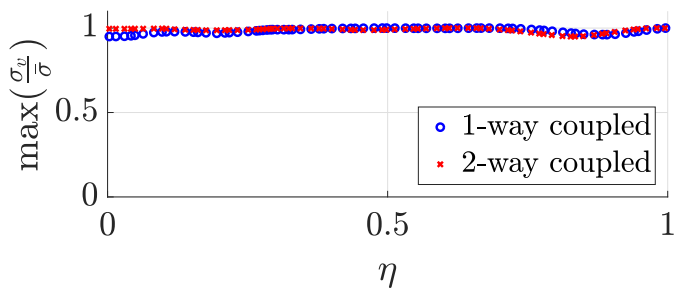

(a) $\beta=1$, straight wall spars

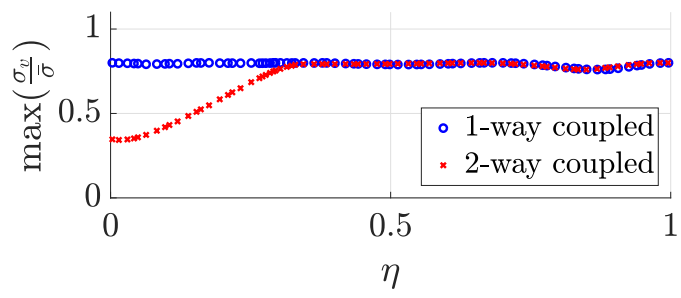

(c) $\beta=0.8$, straight wall spars

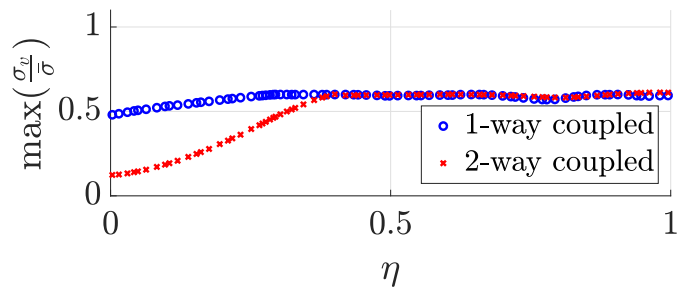

(e) $\beta=0.6$, straight wall spars

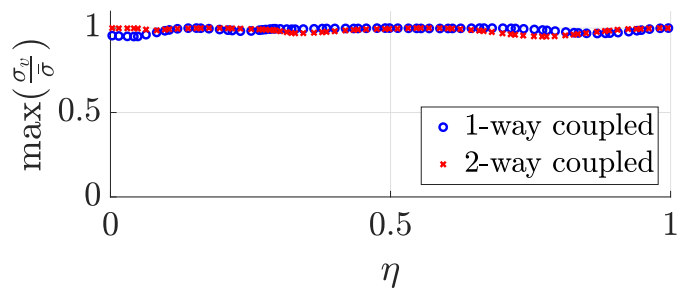

(b) $\beta=1$, curved wall spars

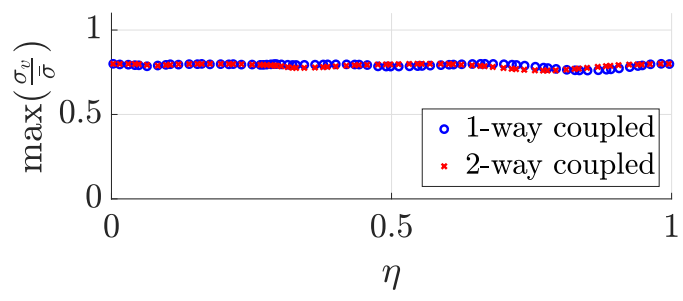

(d) $\beta=0.8$, curved wall spars

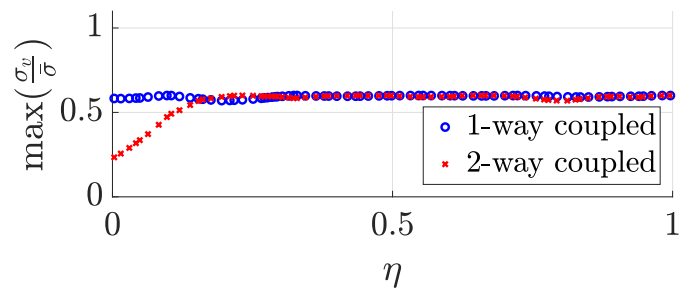

(f) $\beta=0.6$, curved wall spars

Fig. 12 Maximum stress ratio at sections along the beam for optimized designs with 1- and 2-way coupling. 


\begin{tabular}{lcc}
\hline spar walls & $\beta$ & $\Delta_{m}[\%]$ \\
\hline straight & 1 & 21.8 \\
straight & 0.8 & 148.7 \\
straight & 0.6 & 130.0 \\
curved & 1 & 21.7 \\
curved & 0.8 & 40.4 \\
curved & 0.6 & 79.8 \\
\hline
\end{tabular}

Table 1 Difference in mass, $\Delta_{m}$ between 1-way coupled to 2-way coupled designs, for both straight and curved wall spars.

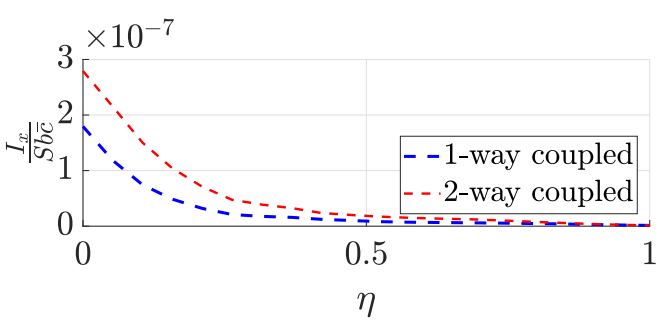

(a) $\beta=1$, bending stiffness

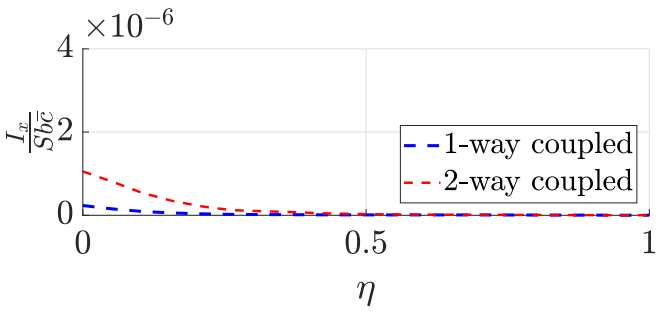

(c) $\beta=0.8$, bending stiffness

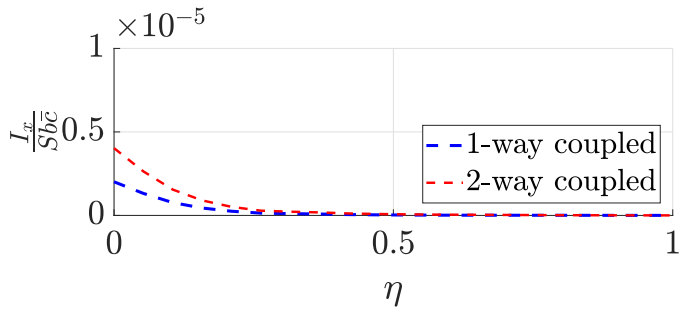

(e) $\beta=0.6$, bending stiffness

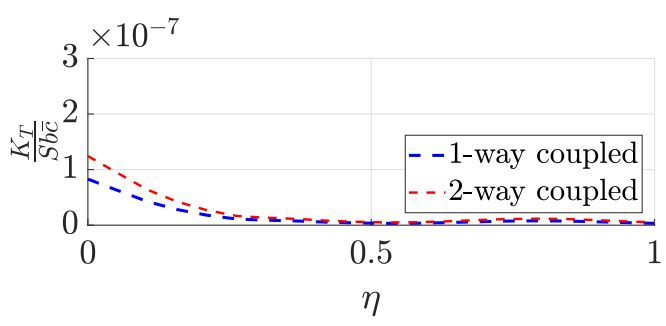

(b) $\beta=1$, torsional stiffness

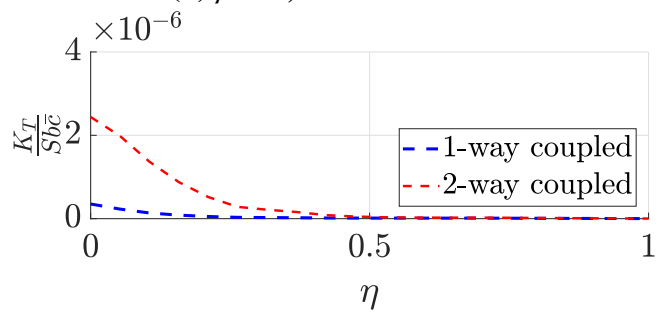

(d) $\beta=0.8$, torsional stiffness

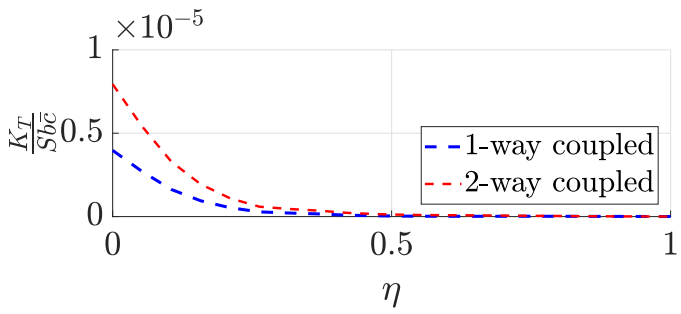

(f) $\beta=0.6$, torsional stiffness

Fig. 13 Stiffness properties for straight wall spar designs in primary loading with different $\beta$ values.

For $\beta=1$ the maximum stress distribution is constant along the length of the wing regardless of coupling method or spar definition. When a stricter constraint is enforced on the stresses the 1-way coupled designs appear to have similar stress distributions along the wing. However, for 2-way coupled designs the stresses are reduced towards the root due to an increased stiffness in that region (refer to Fig. 13). By increasing the stiffness at the root, the deflections along the wing are reduced. The increased stiffness means that the stress constraint is not active at the root, but by reducing the deflections, the loading is reduced and the stress constraint can be satisfied in the outer regions of wing. The onset of this occurs for higher $\beta$ values with straight wall spars, as the curved wall spars allow a larger torsional stiffness which 
can be used to reduce stresses in the outer portions of the wing. This allows the design to satisfy the stress constraint without as much material placed at the root to restrict deformations. The additional stiffness does however result in more mass to be added at the root of the wing leading to large differences in the objective function between 1- and 2-way coupled designs, as shown in Table 1 . When stress constraints are relaxed, i.e. $\beta=1$, there is still a difference of $21 \%$ in the objective function between 1- and 2-way coupled designs. This study demonstrates the importance of solving these problems in the deformed configuration, i.e. using a 2-way coupled method. This finding aligns with previous studies in the literature, where different physics models were used [11, 39, 40].

\section{B. Multidisciplinary shape optimization}

Finally, we consider the case where the external geometry is defined by design variables using the NACA 5-digit airfoil parametrization, and the beam sections are also allowed to vary, i.e. $\mathbf{d}=\{\boldsymbol{c}, \boldsymbol{t}, \boldsymbol{p}, \boldsymbol{\alpha}, \boldsymbol{w}, \boldsymbol{h}, \boldsymbol{a}, \boldsymbol{s}, \boldsymbol{e}\}$. Consider the optimization problem defined in 24] with the objective of minimizing drag in cruise conditions $\left(\theta=0^{\circ}\right)$.

$$
\begin{aligned}
& \min _{\mathbf{d} \in \mathbb{R}^{N_{\mathrm{d}}}} \quad: D(\boldsymbol{\mu}, \mathbf{U}, \mathbf{d}) \\
& \text { subject to } \quad: L(\boldsymbol{\mu}, \mathbf{U}, \mathbf{d})=W_{T}(\mathbf{d}) \\
& \hat{\sigma}_{i}(\boldsymbol{\mu}, \mathbf{U}, \mathbf{d})-\bar{\sigma} \leq 0 \quad \text { for } i=1, \ldots, N_{r} \\
& M_{p}(\boldsymbol{\mu}, \mathbf{U}, \mathbf{d})=0 \\
& \underline{d} \leq d_{i} \leq \bar{d} \quad \text { for } i=1, \ldots, N_{\mathrm{d}}
\end{aligned}
$$

In order to satisfy equilibrium in cruise, the total lift must be equal to the aircraft weight. This is enforced by a constraint, where the weight is defined as the sum of the wingbox structure, $W_{b}$, a payload, $W_{p}$, and $5 \%$ of the wing volume to account for skin thickness, ribs and additional stiffeners, $W_{v}$.

$$
W_{T}(\mathbf{d})=W_{b}(\mathbf{d})+W_{v}(\mathbf{d})+W_{p}
$$

The optimization problem is also subject to stress constraints at an additional load case with a pitch angle, $\theta=10^{\circ}$, simulating a high lift condition. As only the lift-induced drag is considered, the optimizer will seek to reduce lift in order to reduce the drag. This in-turn will require the weight to be reduced due to the first constraint on lift-weight equilibrium. The weight is bounded by the stress constraint because if the weight continues to reduce, the beam will experience large strains leading to large stresses. The final constraint is on the pitching moment in cruise which must be equal to zero. The pitching moment is calculated about a point mid-way downstream of quarter chord axis (refer to Fig. 1). We note that the pitching moment constraint can be neglected and the problem will still converge to reasonable designs. However, the pitching moment is directly related to the airfoil camber and without this additional consideration 
the optimizer could use twist, camber or any combination of two to produce the desired lift for a minimum drag. This creates many possible solutions to the optimization problem, thus the pitching moment constraint offers a means to include an additional practical consideration whilst making the optimization problem better posed.

Sweep is used in aircraft wings to delay the formation of shock waves in transonic flow, a phenomena which cannot be predicted by the panel method. It is also used to ensure stability which is of particular importance in flying wing configurations. Here, optimization problems are solved for fixed sweep angles $\Lambda=\left\{0^{\circ}, 15^{\circ}, 30^{\circ}\right\}$ where converged designs are shown in Fig. 14 The loading for converged designs are shown in Fig. 15, with geometry and performance details tabulated in Table 2 .
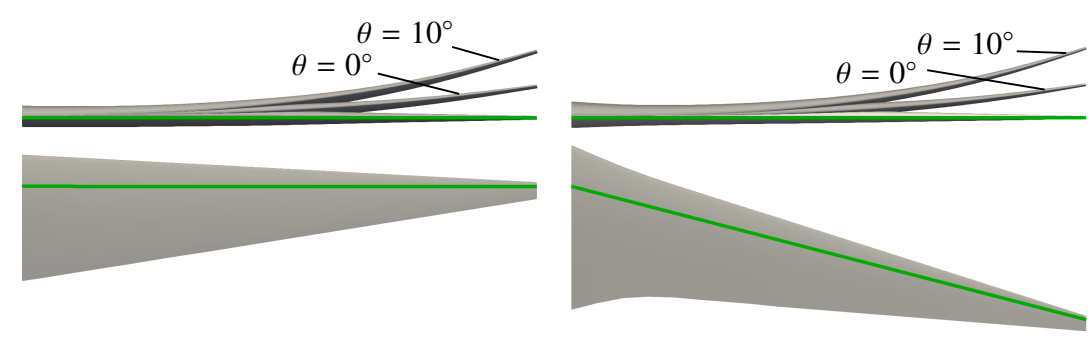

(a) $\Lambda=0^{\circ}$

(b) $\Lambda=15^{\circ}$

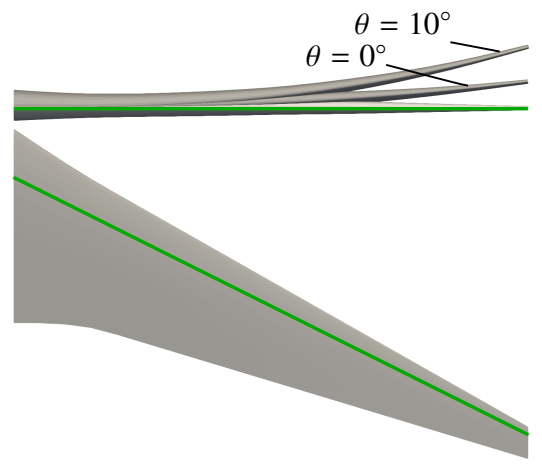

(c) $\Lambda=30^{\circ}$

Fig. 14 Planform and deflections for converged designs with different sweep angles, $\Lambda$. Beam axis shown in green.

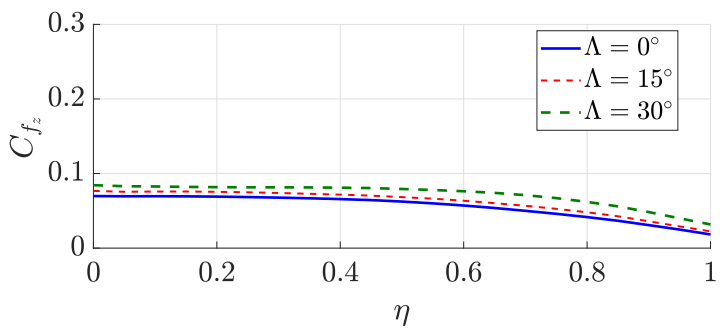

(a) $\theta=0^{\circ}$

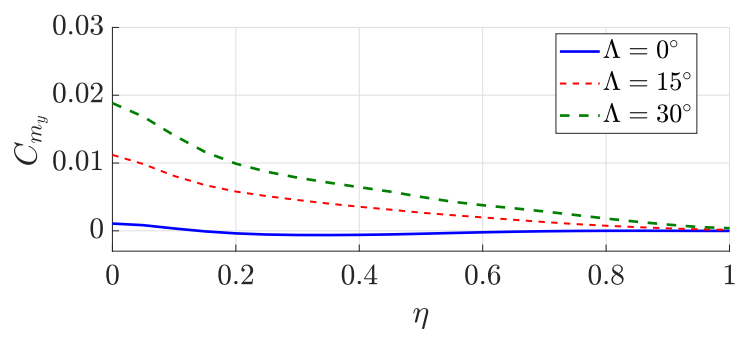

(c) $\theta=0^{\circ}$

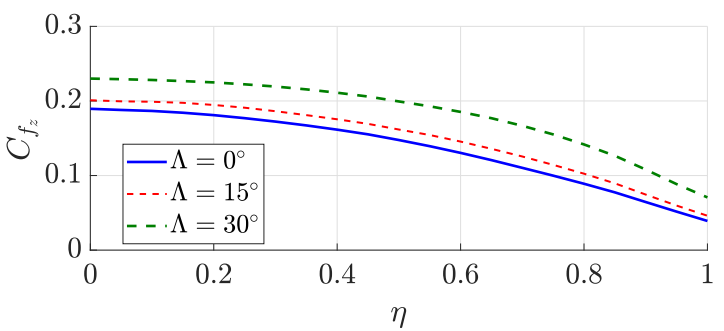

(b) $\theta=10^{\circ}$

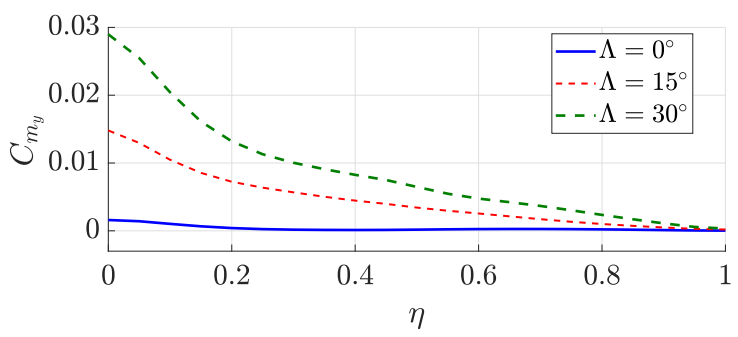

(d) $\theta=10^{\circ}$

Fig. 15 Primary beam loading for converged designs in cruise and high load cases: (a)-(b) normal load coefficient, (c)-(d) torsional load coefficient.

All designs produce high lift to drag ratios between 55 and 60 which is expected as induced drag is the only type of 


\begin{tabular}{lccc}
\hline$\Lambda$ & $R$ & $\frac{U_{t}}{b}[\%]$ & $\frac{L}{D}$ \\
\hline $0^{\circ}$ & 14.5 & 6.72 & 59.1 \\
$15^{\circ}$ & 12.1 & 6.45 & 57.6 \\
$30^{\circ}$ & 9.9 & 5.96 & 55.8 \\
\hline
\end{tabular}

Table 2 Aspect ratio, tip deflection and lift to drag ratio for converged designs with different sweep angles, $\Lambda$.

drag considered. The lift to drag ratio steadily decreases with increasing sweep. Each design converges to large aspect ratio wings which is expected as the most effective way to minimize induced drag is to increase aspect ratio. Designs also have large taper ratios which is motivated by the structural model in order to reduce deformations. This also leads to a large chord length at the root, particularly in swept wings where the loading is high. The stiffer structures that arise in larger swept wings create reductions in the tip displacement, $U_{t}$. The airfoil sections at the root, mid-section, and tip for each design are shown in Fig. 16

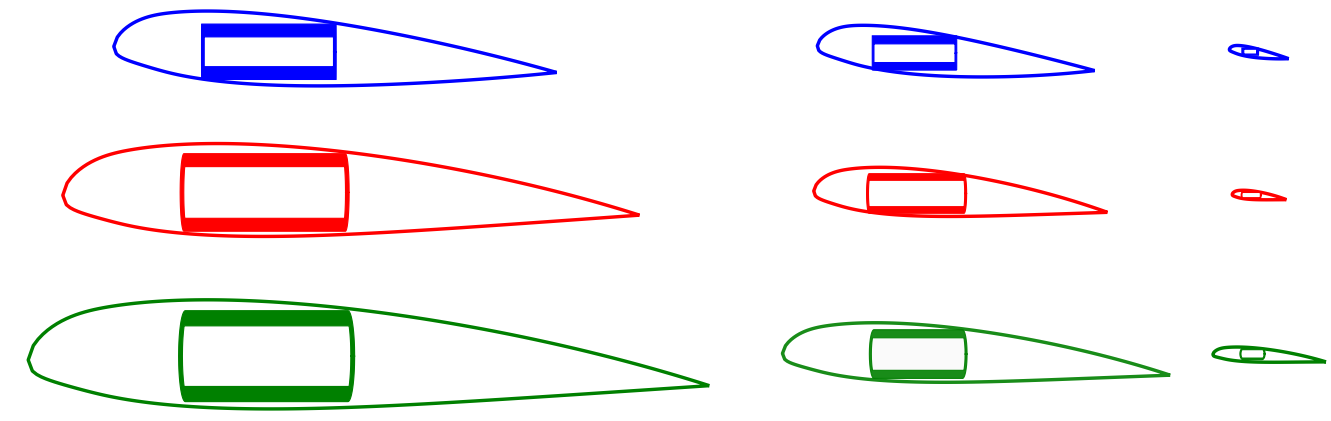

Fig. 16 Cross sections at root, mid-span, and tip (left to right) for $\Lambda=0^{\circ}$ (top), $\Lambda=15^{\circ}$ (middle), $\Lambda=30^{\circ}$ (bottom).

The airfoil parametrization allows a reflex camber line to form in order to aid the fulfillment of the pitching moment constraint. However, only the unswept wing used a reflex camber-line. The swept wings experience higher torsional loads leading to larger twist deformations and the pitching moment constraint to be satisfied in the deformed configuration.

The maximum stress ratio per section is plotted in Fig. 17 for each of the optimized designs. When there is no sweep, the point where the pitching moment is calculated is near-coincident to the beam axis, meaning the pitching moment constraint pushes the torsional loads to near zero (refer to 15). Without torsional loads the optimized design does not converge with curved wall spars as to do so will reduce bending stiffness (Fig. 16. Also, the bending stresses tend to zero towards the tip of the wing, so with low torsional stresses the maximum von Mises stress will also tend to zero towards at the tip as seen in Fig. 17. Stress distributions for the swept wing cases are similar, as are their cross sections (Fig. 16) and loading (Fig. 15). However the loading increases with increasing sweep which requires more stiffness and a larger wingbox. As stated previously, the stiffness distributions give more of an insight into the properties 
of the beam, these are shown in Fig. 18. Compared to the CRM wing results in Section IV.A. the curved wall spars of these design are more moderate because torsional loads are lower. There is also a larger difference between the flange and spar thicknesses of these designs because bending loads are more dominant. But this difference in wall thickness also means torsional stiffness can be reduced for large-curved spar walls (refer to appendix A).

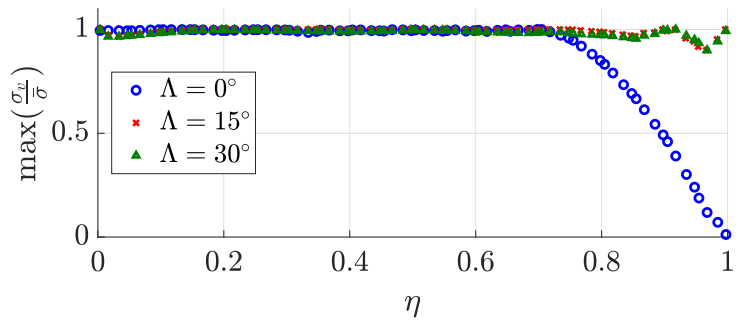

Fig. 17 Maximum stress-ratio in the beam cross-section plotted along the wing for different sweep angles.

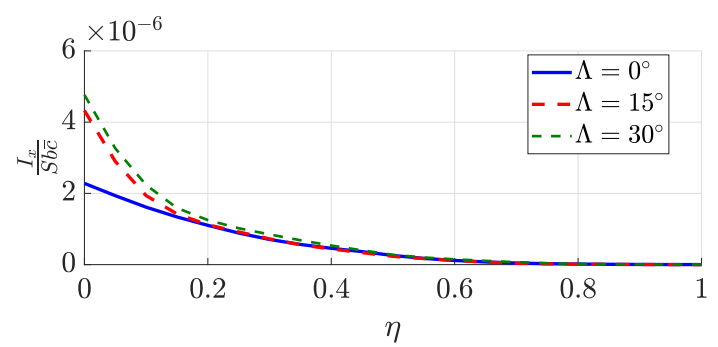

(a)

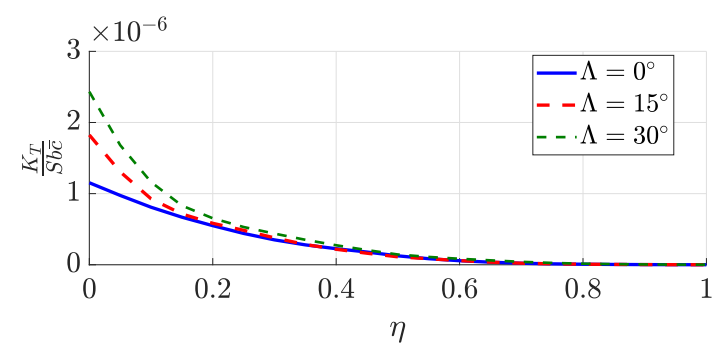

(b)

Fig. 18 Bending and torsional stiffness distributions for optimized designs with different sweep angles.

As a comparison we consider the case where $\Lambda=15^{\circ}$ and the problem is solved using 1-way coupling. Planform and airfoil sections for the optimized design is shown in Fig. 19. When 1-way coupling is used the design has similar planform to that of 2-way coupled design. However, there are more differences in the cross sections. The twist is reduced towards the tip in order to satisfy the pitching moment constraint in the undeformed configuration. Due to reductions in twist and the 1-way coupling, the torsional loads are lower in this case leading to less-curved spar walls and a shorter width in the wing box ( $w$ in Fig. 3). This also provides a reduction in mass, which leads to a lower lift through the equilibrium constraints, and a lower lift-induced drag. From lifting line theory, we know the induced drag is proportional to the square of lift, so the drag will reduce at a faster rate than the lift. This allows the 1-way coupled design to increase the lift to drag ratio to 61.3 .

\section{Conclusion}

The current work introduces a framework for solving aeroelastic optimization problems based on coupled 3D panel and beam finite element methods, with a generalized load-displacement transfer scheme. The framework has proven to work effectively in producing optimized designs with mid-fidelity physics models. Straight and curved wall spars are 


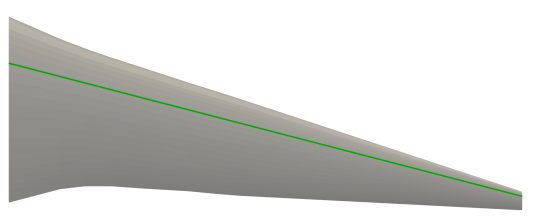

(a)

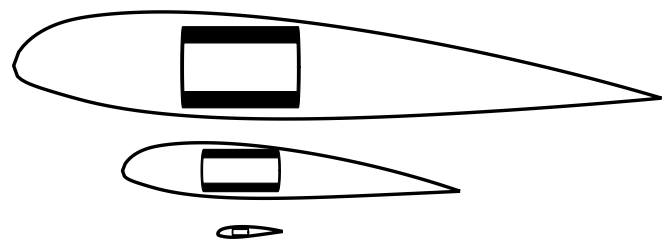

(b)

Fig. 19 Optimized design for 1-way coupling with $\Lambda=15^{\circ}$ : (a) Planform with beam axis shown in green, (b) cross sections at root (top), mid-span (middle), and tip (bottom).

compared based on their performance in compliance minimization and stress-constrained problems. In both cases, the curved wall spars have proven to achieve better designs as they offer more design freedom to create trade-offs between torsional and bending stiffness. This confirms the predictions presented in [27] where curved wall spars first appeared. Comparisons are presented between 1- and 2-way coupling methods where it is shown that the change in loading for deformed configurations can have a significant effect on the optimized design and its performance. This highlights the importance of calculating loads in the deformed configuration and solving the aeroelastic problem to convergence. The current beam parametrization method represents a simplified wingbox that is used to demonstrate the capabilities of the MDO framework and arrive at the conclusions previously stated. However, the methods presented here can easily be extended to a more advanced cross-sectional parametrization and analysis for sectional stiffness properties. Alternatively, the current parametrization method is robust enough to allow a large variance in stiffness properties along the wing whilst remaining physical. These span-wise stiffness distributions can be realized post-optimization using more common aerospace structures such as spar and rib configurations. 


\section{Appendices}

\section{A. Effect of curved spar walls}

Consider a single beam cross section parameterized as described in Section II.C. Fig. 20 plots the relative difference in stiffness properties, $\Delta$, when spar walls become increasingly more curved. The spar walls are straight for $e / h=0$ and semi-circular for $e / h=0.5$. The torsional stiffness for thin walled sections is defined as

$$
K_{T}=\frac{4 A_{s}^{2}}{\oint \frac{d s}{t\left(x_{b}, z_{b}\right)}}
$$

where $s$ is the perimeter of the wingbox defined by the wall's centerline, $A_{s}$ is the area enclose by $s$, and $t$ is the wall thickness.

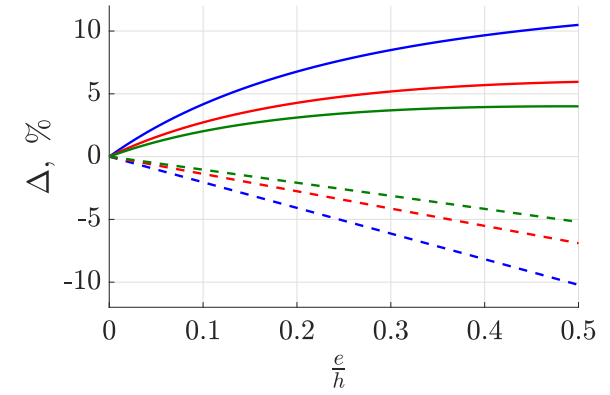

(a) $\frac{a}{s}=1$

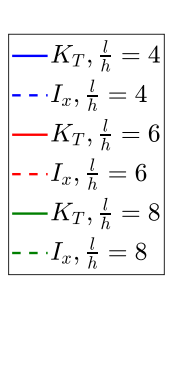

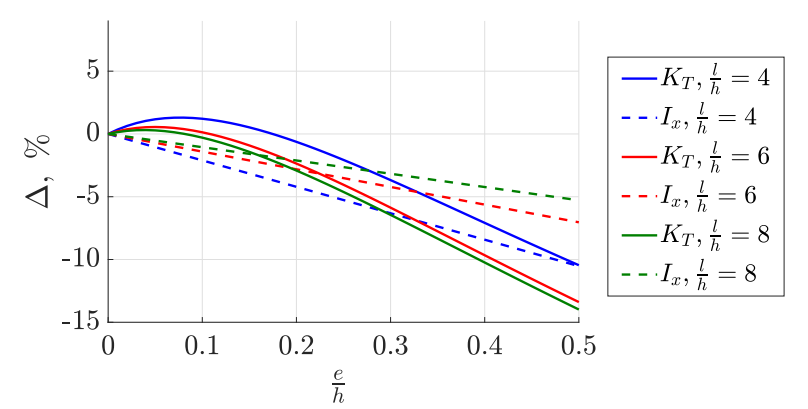

(b) $\frac{a}{s}=10$

Fig. 20 Effect on torsional and bending stiffness by curving the spar walls for two different spar-flange thickness ratios.

Increasing the curvature of the spar walls can lead to increased torsional stiffness but reductions in bending stiffness. For a constant wall thickness the torsional stiffness is proportional to the square of the cross-sectional area over the perimeter. This is why cylinders represents the optimal design for purely torsional loads, and why we see an increase in torsional stiffness for curved wall spars. However, these relationships can also vary with the differences in thickness between spars and flanges, where it is less advantageous for common thick-flange, thin-webbed beam structures. This is due to the beam parametrization method as when the spar walls curve some material is also removed from the flanges. If the flanges are thicker than the spars, then by equation $[26$, the thickness of the flange can have a larger contribution to the torsional stiffness. In this way, large-curved spar walls will remove more material from the flange leading to reductions in torsional stiffness. 


\section{B. Stress analysis}

Four stress states exist the beam: longitudinal stress; torsional stress; and shear stress in the two transverse directions. These stresses are defined for a thin-walled Timoshenko beam as follows

$$
\begin{gathered}
\sigma_{L}=E\left(\varepsilon_{y}+\kappa_{z} x_{b}-\kappa_{x} z_{b}\right) \\
\tau_{T}=\frac{G K_{T}}{2 A t\left(x_{b}, z_{b}\right)} \kappa_{y} \\
\tau_{F_{x}}=\frac{G A Q_{z}\left(x_{b}, z_{b}\right)}{I_{z} t\left(x_{b}, z_{b}\right)} \varepsilon_{x} \\
\tau_{F_{z}}=\frac{G A Q_{x}\left(x_{b}, z_{b}\right)}{I_{x} t\left(x_{b}, z_{b}\right)} \varepsilon_{z}
\end{gathered}
$$

where $x_{b}$ and $z_{b}$ are local coordinates on the cross section at the point of stress evaluation, $t$ is the local thickness, and $Q$ is the first moment of area.

In the assumptions of thin-walled cross sections the shear direction is defined by the shear flow, and the total shear, $\tau_{x}$ and $\tau_{z}$, is the sum of the individual contributions, $\tau_{T}, \tau_{F_{x}}$, and $\tau_{F_{z}}$ [34]. The shear flow diagrams for our cross section are shown in Figs. 21:21. The maximum von Mises stress can occur at a possible 16 locations in a given cross section (shown in Fig. 21 d). The exact location will depend on the loading conditions of the beam. Von Mises stress is calculated at each of these 16 locations in the cross sections via

$$
\sigma_{v}=\sqrt{\sigma_{y}^{2}+3\left(\tau_{x}^{2}+\tau_{z}^{2}\right)}
$$

\section{Sensitivity analysis}

All sensitivities are calculated via a discrete adjoint method. Consider the objective/constraint function $f$ expressed in augmented Lagrangian form as

$$
\mathcal{F}=f(\boldsymbol{\mu}, \mathbf{U}, \mathbf{d})+\lambda_{a}^{\mathrm{T}} \mathbf{R}_{a}(\boldsymbol{\mu}, \mathbf{U}, \mathbf{d})+\lambda_{b}^{\mathrm{T}} \mathbf{R}_{b}(\boldsymbol{\mu}, \mathbf{U}, \mathbf{d})
$$

where $\lambda_{a}$ and $\lambda_{b}$ are Lagrangian multipliers for aerodynamic and beam models respectively. The total derivative of $\mathcal{F}$ with respect to $\mathbf{d}$ is derived to be

$$
\frac{d \mathcal{F}}{d \mathbf{d}}=\frac{\partial f}{\partial \mathbf{d}}+\lambda_{a}^{\mathrm{T}}\left[\frac{\partial \mathbf{A}}{\partial \mathbf{d}} \boldsymbol{\mu}+\frac{\partial \mathbf{B}}{\partial \mathbf{d}} \boldsymbol{\sigma}+\mathbf{B} \frac{\partial \boldsymbol{\sigma}}{\partial \mathbf{d}}\right]+\lambda_{b}^{\mathrm{T}}\left[\frac{\partial \mathbf{K}}{\partial \mathbf{d}} \mathbf{U}-\frac{\partial \mathbf{P}}{\partial \mathbf{d}}\right]
$$




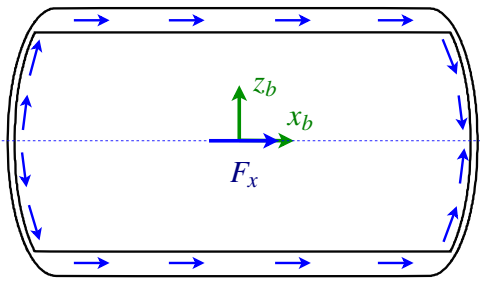

(a) Shear flow for shear force, $F_{x}$

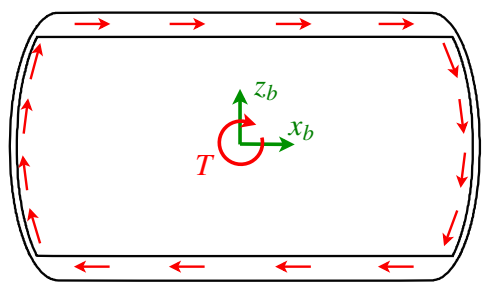

(c) Shear flow for torsion, $T$

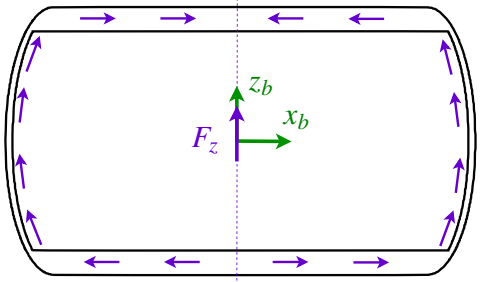

(b) Shear flow for shear force, $F_{z}$

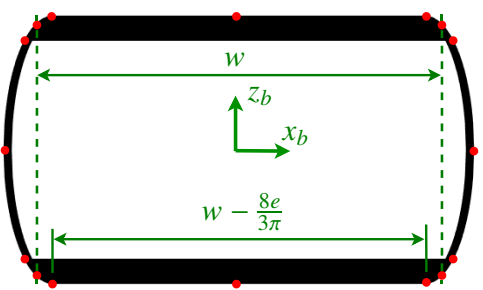

(d) Stress evaluation points

Fig. 21 Methods for stress evaluation.

where the operator $\frac{\partial}{\partial \mathbf{d}}$ captures only explicit dependence on $\mathbf{d}$, and $\lambda_{a}$ and $\lambda_{b}$ are calculated through solving the following adjoint equations

$$
\begin{gathered}
\lambda_{a}^{\mathrm{T}} \mathbf{A}-\lambda_{b}^{\mathrm{T}} \frac{\partial \mathbf{P}}{\partial \boldsymbol{\mu}}=-\frac{\partial f}{\partial \boldsymbol{\mu}} \\
\lambda_{a}^{\mathrm{T}}\left[\frac{\partial \mathbf{A}}{\partial \mathbf{U}} \boldsymbol{\mu}+\frac{\partial \mathbf{B}}{\partial \mathbf{U}} \boldsymbol{\sigma}+\mathbf{B} \frac{\partial \boldsymbol{\sigma}}{\partial \mathbf{U}}\right]+\lambda_{b}^{\mathrm{T}}\left[\mathbf{K}-\frac{\partial \mathbf{P}}{\partial \mathbf{U}}\right]=-\frac{\partial f}{\partial \mathbf{U}}
\end{gathered}
$$

or

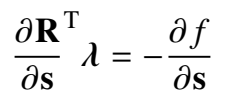

with $\lambda=\left\{\lambda_{a} \lambda_{b}\right\}^{\mathrm{T}}$.

\section{Funding Sources}

This work is funded by the Villum Foundation under the InnoTop VILLUM investigator project.

\section{References}

[1] Haftka, R. T., "Optimization of flexible wing structures subject to strength and induced drag constraints," AIAA Journal, Vol. 15, No. 8, 1977, pp. 1101-1106. doi:https://doi.org/10.2514/3.7400.

[2] Grossman, B., Strauch, G. J., Eppard, W. M., Gurdal, Z., and Haftka, R. T., "Integrated aerodynamic/structural design of a sailplane wing," Journal of Aircraft, Vol. 25, No. 9, 1988, pp. 855-860. doi:https://doi.org/10.2514/3.45670.

[3] Martins, J. R., Alonso, J. J., and Reuther, J. J., "High-fidelity aerostructural design optimization of a supersonic business jet," Journal of Aircraft, Vol. 41, No. 3, 2004, pp. 523-530. doi:https://doi.org/10.2514/1.11478. 
[4] Barcelos, M., and Maute, K., "Aeroelastic design optimization for laminar and turbulent flows," Computer Methods in Applied Mechanics and Engineering, Vol. 197, No. 19-20, 2008, pp. 1813-1832. doi:https://doi.org/10.1016/j.cma.2007.03.009.

[5] Kenway, G. K., Kennedy, G. J., and Martins, J. R., "Scalable parallel approach for high-fidelity steady-state aeroelastic analysis and adjoint derivative computations," AIAA Journal, Vol. 52, No. 5, 2014, pp. 935-951. doi:https://doi.org/10.2514/1.j052255.

[6] Brooks, T. R., Kenway, G. K., and Martins, J. R., "Benchmark aerostructural models for the study of transonic aircraft wings," AIAA Journal, Vol. 56, No. 7, 2018, pp. 2840-2855. doi:https://doi.org/10.2514/1.j056603.

[7] Unger, E. R., Haftka, R. T., Grossman, B., and Mason, W. H., "Efficient optimization of integrated aerodynamic-structural design," International Journal for Numerical Methods in Engineering, Vol. 28, No. 3, 1989, pp. 593-607. doi:https://doi.org/doi: 10.1002/nme.1620280308.

[8] Grossman, B., Polen, D. M., Sobieszczanski-Sobieski, J., Haftka, R. T., Kao, P.-J., and Rais-Rohani, M., "Integrated aerodynamic-structural design of a transport wing," Journal of Aircraft, Vol. 27, No. 12, 1990, pp. 1050-1056. doi: https://doi.org/10.2514/3.45980.

[9] Jasa, J. P., Hwang, J. T., and Martins, J. R., "Open-source coupled aerostructural optimization using Python,” Structural and Multidisciplinary Optimization, Vol. 57, No. 4, 2018, pp. 1815-1827. doi:https://doi.org/10.1007/s00158-018-1912-8.

[10] Chauhan, S., Shamsheer, and Martins, R. R. A., Joaquim, "Low-fidelity aerostructural optimization of aircraft wings with a simplified wingbox model using OpenAeroStruct," Engopt 2018 Proceedings of the 6th International Conference on Engineering Optimization, 2018, pp. 418-431. doi:https://doi.org/10.1007/978-3-319-97773-7_38.

[11] Dunning, P. D., Stanford, B. K., and Kim, H. A., "Coupled aerostructural topology optimization using a level set method for 3D aircraft wings," Structural and Multidisciplinary Optimization, Vol. 51, No. 5, 2015, pp. 1113-1132. doi:https: //doi.org/10.1007/s00158-014-1200-1.

[12] Vassberg, J. C., DeHaan, M. A., Rivers, S. M., and Wahls, R. A., "Development of a common research model for applied CFD validation studies," Collection of Technical Papers - AIAA Applied Aerodynamics Conference, 2008. doi: https://doi.org/10.2514/6.2008-6919.

[13] Kennedy, G. J., and Martins, J. R. R. A., “A parallel aerostructural optimization framework for aircraft design studies,” Structural and Multidisciplinary Optimization, Vol. 50, No. 6, 2014, pp. 1079-1101. doi:https://doi.org/10.1007/s00158-014-1108-9.

[14] Kennedy, G. J., and Martins, J. R. R. A., "Parallel solution methods for aerostructural analysis and design optimization,” 13th AIAA/ISSMO Multidisciplinary Analysis and Optimization Conference, American Institute of Aeronautics and Astronautics Inc., 2010. doi:https://doi.org/10.2514/6.2010-9308.

[15] Kennedy, G. J., and Martins, J. R. R. A., "A comparison of metallic and composite aircraft wings using aerostructural design optimization," 12th AIAA Aviation Technology, Integration and Operations (ATIO) Conference and 14th AIAA/ISSMO Multidisciplinary Analysis and Optimization Conference, American Institute of Aeronautics and Astronautics Inc., 2012. doi:https://doi.org/10.2514/6.2012-5475. 
[16] James, K. A., Kennedy, G. J., and Martins, J. R. R. A., "Concurrent aerostructural topology optimization of a wing box," Computers and Structures, Vol. 134, 2014, pp. 1-17. doi:https://doi.org/10.1016/j.compstruc.2013.12.007.

[17] Chin, T. W., and Kennedy, G. J., "Large-scale compliance-minimization and buckling topology optimization of the undeformed common research model wing," 57th AIAA/ASCE/AHS/ASC Structures, Structural Dynamics, and Materials Conference, American Institute of Aeronautics and Astronautics Inc., 2016. doi:https://doi.org/10.2514/6.2016-0939.

[18] Goetzendorf-Grabowski, T., and Mieloszyk, J., "Common computational model for coupling panel method with finite element method," Aircraft Engineering and Aerospace Technology, Vol. 89, No. 5, 2017, pp. 654-662. doi:https://doi.org/10.1108/AEAT01-2017-0044.

[19] Mieloszyk, J., and Goetzendorf-Grabowski, T., "Introduction of full flight dynamic stability constraints in aircraft multidisciplinary optimization,” Aerospace Science and Technology, Vol. 68, 2017, pp. 252-260. doi:https://doi.org/10.1016/j.ast.2017. 05.024 .

[20] Kenway, G., and Martins, J. R., "Aerostructural shape optimization of wind turbine blades considering site-specific winds," 12th AIAA/ISSMO Multidisciplinary Analysis and Optimization Conference, MAO, 2008, pp. 2008-6025. doi:https://doi.org/10. 2514/6.2008-6025.

[21] Ashuri, T., Zaaijer, M., Martins, J., van Bussel, G., and van Kuik, G., "Multidisciplinary design optimization of offshore wind turbines for minimum levelized cost of energy,” Renewable Energy, Vol. 68, 2014, pp. 893 - 905. doi:https://doi.org/10.1016/j. renene.2014.02.045.

[22] Zahle, F., Tibaldi, C., Pavese, C., McWilliam, M., Blasques, J. P. A. A., and Hansen, M. H., "Design of an aeroelastically tailored 10 MW wind turbine rotor," Journal of Physics: Conference Series (online), Vol. 753, No. 6, 2016 , p. 062008. doi:10.1088/1742-6596/753/6/062008.

[23] Wang, Z., Suiker, A. S., Hofmeyer, H., van Hooff, T., and Blocken, B., "Coupled aerostructural shape and topology optimization of horizontal-axis wind turbine rotor blades," Energy Conversion and Management, Vol. 212, 2020 , p. 112621. doi:https://doi.org/10.1016/j.enconman.2020.112621.

[24] Sessarego, M., Feng, J., Ramos García, N., and Horcas, S. G., "Design optimization of a curved wind turbine blade using neural networks and an aero-elastic vortex method under turbulent inflow,” Renewable Energy, Vol. 146, 2020, pp. 1524-1535. doi:https://doi.org/10.1016/j.renene.2019.07.046.

[25] McWilliam, M., Lawton, S., and Crawford, C., "Towards a framework for aero-elastic multidisciplinary design optimization of horizontal axis wind turbines," 51st AIAA Aerospace Sciences Meeting including the New Horizons Forum and Aerospace Exposition, ???? doi:https://doi.org/10.2514/6.2013-200.

[26] McWilliam, M. K., “Towards multidisciplinary design optimization capability of horizontal axis wind turbines,” Ph.D. thesis, University of Victoria, 2015. 
[27] Aage, N., Andreassen, E., Lazarov, B. S., and Sigmund, O., "Giga-voxel computational morphogenesis for structural design," Nature, Vol. 550, No. 7674, 2017, pp. 84-86. doi:https://doi.org/10.1038/nature23911.

[28] Conlan-Smith, C., Ramos-García, N., Sigmund, O., and Schousboe Andreasen, C., "Aerodynamic shape optimization of aircraft wings using panel methods," AIAA Journal, Published online May 2020. doi:https://doi.org/10.2514/1.J058979.

[29] Yu, W., Volovoi, V. V., Hodges, D. H., and Hong, X., "Validation of the variational asymptotic beam sectional analysis," AIAA Journal, Vol. 40, No. 10, 2002, pp. 2105-2112. doi:10.2514/2.1545.

[30] Yu, W., Hodges, D. H., and Ho, J. C., "Variational asymptotic beam sectional analysis - An updated version,” International Journal of Engineering Science, Vol. 59, 2012, pp. 40-64. doi:10.1016/j.ijengsci.2012.03.006.

[31] Blasques, J. P. A. A., User's Manual for BECAS: A cross section analysis tool for anisotropic and inhomogeneous beam sections of arbitrary geometry, Risø DTU - National Laboratory for Sustainable Energy, 2012.

[32] Drela, M., Flight vehicle aerodynamics, MIT Press, 2014.

[33] Ramos García, N., Sørensen, J., and Shen, W., "Three-dimensional viscous-inviscid coupling method for wind turbine computations," Wind Energy, Vol. 19, No. 1, 2016, p. 67-93. doi:https://doi.org/10.1002/we.1821.

[34] Parnes, R., Solid mechanics in engineering, Wiley,, 2001.

[35] Giavotto, V., Borri, M., Mantegazza, P., and Ghiringhelli, G., "Anisotropic beam theory and applications," Computers and Structures, Vol. 16, No. 1-4, 1983, pp. 403-413. doi:https://doi.org/10.1016/0045-7949(83)90179-7.

[36] Martins, J. R., Alonso, J. J., and Reuther, J. J., “A coupled-adjoint sensitivity analysis method for high-fidelity aero-structural design," Optimization and Engineering, Vol. 6, No. 1, 2005, pp. 33-62. doi:https://doi.org/10.1023/B:OPTE.0000048536. 47956.62 .

[37] Svanberg, K., "The method of moving asymptotes - a new method for structural optimization,” International Journal for Numerical Methods in Engineering, Vol. 24, No. 2, 1987, pp. 359-373. doi:https://doi.org/10.1002/nme.1620240207.

[38] Le, C., Norato, J., Bruns, T., Ha, C., and Tortorelli, D., "Stress-based topology optimization for continua," Structural and Multidisciplinary Optimization, Vol. 41, No. 4, 2010, pp. 605-620. doi:https://doi.org/10.1007/s00158-009-0440-y.

[39] Maute, K., and Allen, M., "Conceptual design of aeroelastic structures by topology optimization," Structural and Multidisciplinary Optimization, Vol. 27, No. 1-2, 2004, pp. 27-42. doi:https://doi.org/10.1007/s00158-003-0362-z.

[40] Stanford, B. K., and Dunning, P. D., "Optimal topology of aircraft rib and spar structures under aeroelastic loads," Journal of Aircraft, Vol. 52, No. 4, 2015, pp. 1298-1311. doi:https://doi.org/10.2514/1.C032913. 